TOPICAL REVIEW

\title{
Flow-induced structure in colloidal suspensions
}

\author{
J Vermant $^{1}$ and M J Solomon ${ }^{2}$ \\ ${ }^{1}$ Department of Chemical Engineering, K U Leuven, W de Croylaan 46, B-3001 Leuven, \\ Belgium \\ 2 Department of Chemical Engineering, University of Michigan, Ann Arbor, MI 48109-2136, \\ USA
}

Received 14 October 2004

Published 14 January 2005

Online at stacks.iop.org/JPhysCM/17/R187

\begin{abstract}
We review the sequences of structural states that can be induced in colloidal suspensions by the application of flow. Structure formation during flow is strongly affected by the delicate balance among interparticle forces, Brownian motion and hydrodynamic interactions. The resulting nonequilibrium microstructure is in turn a principal determinant of the suspension rheology. Colloidal suspensions with near hard-sphere interactions develop an anisotropic, amorphous structure at low dimensionless shear rates. At high rates, clustering due to strong hydrodynamic forces leads to shear thickening rheology. Application of steady-shear flow to suspensions with repulsive interactions induces a rich sequence of transitions to one-, two-and threedimensional order. Oscillatory-shear flow generates metastable ordering in suspensions with equilibrium liquid structure. On the other hand, shortrange attractive interactions can lead to a fluid-to-gel transition under quiescent suspensions. Application of flow leads to orientation, breakup, densification and spatial reorganization of aggregates. Using a non-Newtonian suspending medium leads to additional possibilities for organization. We examine the extent to which theory and simulation have yielded mechanistic understanding of the microstructural transitions that have been observed.
\end{abstract}

(Some figures in this article are in colour only in the electronic version)

\section{Contents}

1. Introduction 188

2. Effect of flow on amorphous dispersions with near hard-sphere interactions $\quad 190$

2.1. Introduction 190

2.2. Flow distorted microstructure $\quad 190$

$\begin{array}{ll}\text { 2.3. Future prospects } & 193\end{array}$

0953-8984/05/040187+30\$30.00 @ 2005 IOP Publishing Ltd Printed in the UK R187 
3. Effect of flow on the ordering of colloidal suspensions 193

3.1. Introduction 193

3.2. Phase transitions and equilibrium structure 194

3.3. Shear-induced structure in suspensions with hard-sphere and repulsive interactions

3.4. Unresolved issues and future prospects 201

4. Gelling suspensions 202

4.1. Introduction and relevance 202

4.2. Flow-induced structure in aggregated systems 203

4.3. Unresolved issues and challenges 209

5. Suspensions of particles dispersed in viscoelastic media 209

5.1. Introduction and relevance $\quad 209$

5.2. Effects of flow on particles dispersed in viscoelastic liquids 210

$\begin{array}{ll}\text { 6. Conclusions and outlook } & 211\end{array}$

$\begin{array}{ll}\text { References } & 212\end{array}$

\section{Introduction}

Colloidal dispersions are encountered in many consumer products, including paints, inks, cosmetics, pharmaceuticals and food (Hiemenz and Rajagopalan 1997). Colloid science and technology also play a key role in emerging technologies such as, for example, tissue engineering scaffolding (Irvine et al 2003), photonic crystals (Vlasov et al 1999), 3D ink-jet technology (Gratson et al 2004), advanced ceramics processing (Lewis 2000), microfluidics (Terray et al 2002) and nano-composites (Vaia and Giannelis 2001, Solomon et al 2001). The macroscopic properties of suspensions are determined by the spatial organization of the particles, usually referred to as the microstructure. In the absence of flow, a wide range of colloidal organizations can be encountered in different materials, depending on the relative values of Brownian, repulsive and attractive forces (Russel et al 1989, Gast and Russel 1998, Larson 1999, Lowen 2004). When Brownian forces dominate and the volume fraction is below the fluid-solid threshold, the particles organize as in a disordered liquid. When the repulsive forces dominate, ordered crystalline structures can be obtained. At low volume fractions, when the attractive forces dominate, the particles aggregate or gel into scale invariant, selfsimilar clusters with fractal structure (Russel et al 1989, Carpineti and Giglio 1992). At high volume fractions, gels exhibit long-range heterogeneous void structure (Varadan and Solomon 2003a, 2003b, Shah et al 2003). For quiescent suspensions, equilibrium phase behaviour can be predicted by the application of statistical thermodynamics theory and simulation (Russel et al 1989). Although still incomplete, understanding of the quiescent gelation transition has received considerable attention recently (Pham et al 2002, Dawson 2002).

For suspensions subjected to flow, gelation and phase boundaries may depend on the strength of the flow. More importantly, the microstructure rearranges to accommodate the applied hydrodynamic and the colloidal forces. At sufficiently high volume fractions, and when the interactions between colloids are comparable to the forces induced by the hydrodynamics, most types of suspensions display an anisotropic organization when subjected to shear flow (Dhont 1996). The anisotropic microstructure will, in turn, affect the rheological properties, thereby possibly altering the flow profile itself of non-homogeneous fields. Hence understanding the intimate coupling between the flow and the microstructure during flow motivates the work addressed in the present review. The relevance lies in the central role of the microstructure in determining the end-use properties in the above-mentioned application areas, as well as for the understanding and controlling of the processing behaviour of suspensions. 


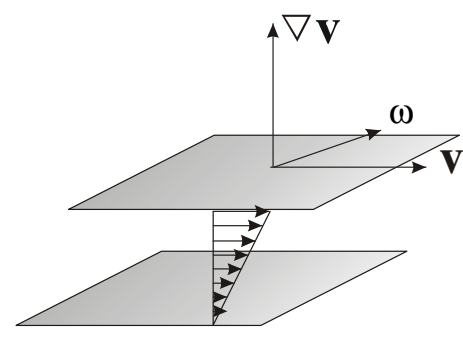

(a)

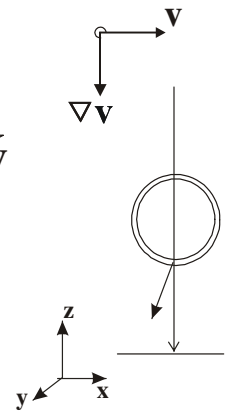

(b)

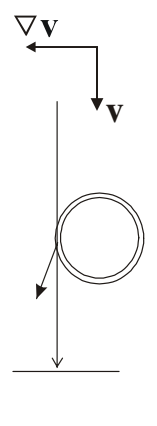

(c)

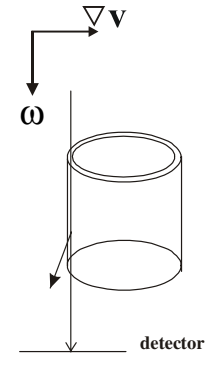

(d)

Figure 1. Typical relative configurations of the shear flow device and scattering geometry for interrogation of shear-induced changes in the structures in colloidal suspensions. (a) Shear flow and definitions of flow $(\mathbf{v})$, velocity gradient $(\nabla \mathbf{v})$ and vorticity $(\omega)$ direction. The scattering vector is observed in the $\mathbf{e}_{x}, \mathbf{e}_{y}$ plane, the incident radiation being along the $\mathbf{e}_{z}$ direction. Typical configurations for a Couette geometry: in (b) scattering vectors with $q_{\nabla}=0$ are probed $(\nabla \mathbf{v}-$ $\omega$ plane), whereas in (c) off-axis incident propagation yields a non-zero component of $q_{\nabla}$; for (d) propagation along the vorticity axis yields a projection of the structure in the $\mathbf{v}-\nabla \mathbf{v}$ plane, and scattering vectors with $q_{\omega}=0$.

In order to investigate these problems, time resolved, in situ experimental techniques have been developed to interrogate the structure in both real and reciprocal space. Microscopy techniques combined with quantitative image analysis directly probe the organization of the colloids in space. Here the two-body structure can be represented by the pair distribution function $g(\mathbf{r})$ (Russel et al 1989). Reciprocal-space techniques typically rely on measuring changes in 2D scattering patterns, using various sources of electromagnetic radiation, i.e. light and $\mathrm{x}$-rays or neutron scattering. Generally the scattered intensity depends on the angle between the incoming ray and the scattered ray, as specified by the scattering vector, $\mathbf{q}$. By probing the directional dependency of the scattered radiation the structure factor $S(\mathbf{q})$ can be measured. $S(\mathbf{q})$ is related to the real-space information $g(\mathbf{r})$ by means of a Fourier transform. Figure 1, after Chen et al (1994a), depicts the relationship between coordinate systems describing the scattering geometry $\left(\mathbf{e}_{x}, \mathbf{e}_{y}, \mathbf{e}_{z}\right)$ and the shear flow $\left(\mathbf{e}_{v}, \mathbf{e}_{\nabla}, \mathbf{e}_{\omega}\right)$ for the particular case of rotational Couette flow. In a small-angle limit the two-dimensional detector probes scattering vectors, $\mathbf{q}$, that reside in the $x-y$ plane. For incident radiation propagating along the centreline of the shear cell (figure 1(b)), this corresponds to measurements with component of the scattering vector in the gradient direction, $q_{\nabla}$, of zero magnitude; hence the structure factor is projected in the $v-\omega$ plane. For incident radiation travelling along the flow direction, as in figure 1(c), this corresponds to measurements with component of the scattering vector in the velocity direction, $q_{\mathbf{v}}$, of zero magnitude. In figure $1(\mathrm{~d})$ the radiation is sent down along the vorticity direction. Scattering measurements using this set-up allow both deformation and reorientation of the structure to be monitored. Measurements of the $S(\mathbf{q})$ projected in the $\mathbf{v}-\nabla \mathbf{v}$ plane are most relevant for understanding the link with the shear and time-dependent viscosity, yet they are the most difficult to perform.

Apart from experimental work, both theoretical and numerical approaches have been used. The major difficulty is to account correctly for the hydrodynamic interactions (see for example Russel et al (1989), Dhont (1996) for an overview of analytical theories). To correctly account for multibody hydrodynamic interactions, an important tool in this respect is the Stokesian dynamics technique, which correctly accounts for hydrodynamic interaction of particles in close contact (Brady and Bossis 1988, Ball and Melrose 1995, Foss and Brady 2000, Banchio and Brady 2003). 
This review is organized as follows. First we start with a brief review of the effects of flow on amorphous suspensions, a subject in which a rather complete understanding of the effects of flow has been achieved. Three sections deal with more recent work, concerning the effects of flow on ordering suspensions, gelling suspensions and suspensions in viscoelastic media.

\section{Effect of flow on amorphous dispersions with near hard-sphere interactions}

\subsection{Introduction}

Below a critical volume fraction, dispersions of Brownian particles with near hard-sphere interactions display a liquid-like structure at equilibrium. When the convective forces associated with for example shear flow dominate the forces associated with Brownian motion, the microstructure is distorted and the asymmetry of the flow field is reflected in the suspension microstructure. Distortion of the microstructure will occur when the timescale associated with flow is smaller than the timescale associated with local scale diffusion, i.e. when the reciprocal of the shear rate is smaller than the time for diffusion $\left(\dot{\gamma}^{-1}<t_{\mathrm{d}}\right)$. Using the Stokes-Einstein relation for the diffusivity, this constraint leads to the well-known dimensionless Péclet number (see for example Krieger and Choi 1982, Russel et al 1989) scaling for the onset of structural distortion:

$$
P e=\frac{\eta_{\mathrm{m}} a^{3} \dot{\gamma}}{k_{\mathrm{B}} T}
$$

Here $a$ is the particle radius, $k_{\mathrm{B}}$ is Boltzman's constant, $T$ is the temperature, $\dot{\gamma}$ is the shear rate and $\eta_{\mathrm{m}}$ is the medium viscosity. When the volume fraction increases, an effective, suspension viscosity better replaces the medium viscosity, because it is the 'effective' medium that on average affects the particle motion. Hence a dimensionless shear stress is appropriate to separate weak and strong flow regimes in concentrated suspensions (Choi and Krieger 1986). At $P e>1$, a distortion of the liquid-like structure can be expected. Experimental and theoretical work has aimed to determine the link between the distortion of the microstructure and the stress tensor, as discussed below. Theoretical work in conjunction with computer simulations have also yielded detailed results about how the hydrodynamic forces influence the suspension microstructure and in turn determine the macroscopic behaviour, especially with respect to the so-called shear-thickening behaviour which occurs in suspensions.

\subsection{Flow distorted microstructure}

The Péclet number (or the dimensionless stress) determines a number of structural transitions. At low $P e$ numbers $(P e \ll 1)$, the microstructure is isotropic, and light scattering experiments, for example, reveal a ring-like (Debye-Scherrer) pattern (Ackerson and Clark 1984, Ackerson 1990, Wagner and Russel 1990). As the Péclet number is increased, the microstructure becomes distorted, as particles are pushed together along the compression axis of the flow field, while being further separated along the extensional axis, as is schematically depicted in figure 2 for the structure in reciprocal space. The shear deformation of the colloidal liquid structure is a smooth process with no long-range string or layer ordering (Wagner and Russel 1990, Yan and Dhont 1993). The distortion of the microstructure is directly related to the changes in the macroscopic properties. A non-equilibrium statistical mechanical analysis of the microstructure in weak flows has been used to derive rheological predictions (Russel and Gast 1986, Wagner and Russel 1990, Adriani and Gast 1989, Dhont 1989). As for molecular fluids (Hess and Hanley 1983a, 1983b), an expansion of the non-equilibrium structure factor in spherical harmonics establishes formal relationships between the structure factor, stress tensor, optical dichroism and birefringence (Wagner and Ackerson 1992). Likewise, the scattering 


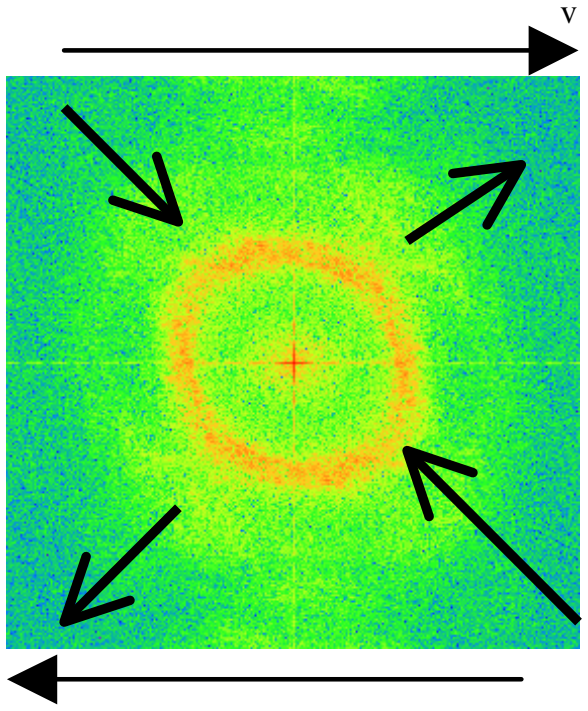

Figure 2. Schematic of the changes in the suspension microstructure as characterized by an FFT of an initially disordered suspension in the $\mathbf{v}-\nabla \mathbf{v}$ plane at high $P e$ number. The arrows on the FFT indicate the compressional axis and the extensional axis of the flow field; the velocity vectors are drawn as well.

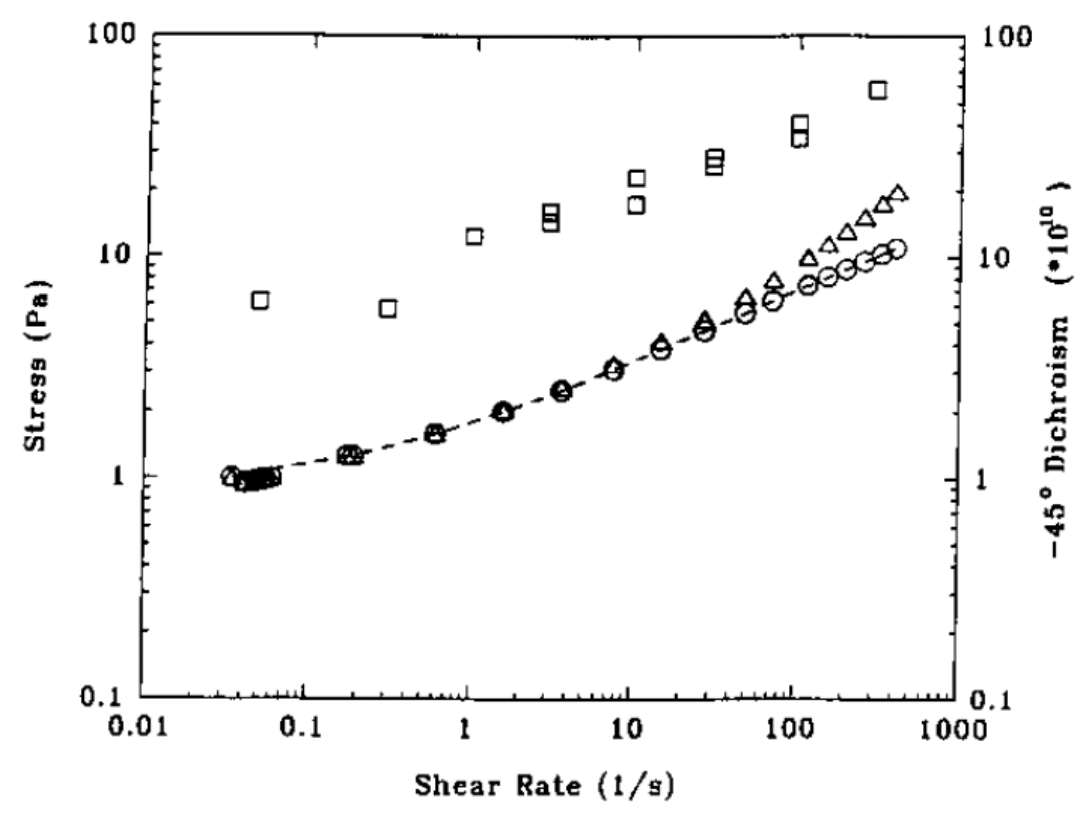

Figure 3. Scattering dichroism $(\square)$, total shear stress $(\triangle)$ and calculated thermodynamic stress $(O)$ for a suspension $(d=180 \mathrm{~nm})$ of polymer-stabilized silica spheres dispersed in THF (reprinted from Bender and Wagner (1995), (C) (1995), with permission).

dichroism arises as a consequence of the distortion of the structure factor (Wagner et al 1988). Bender and Wagner have derived a stress optical relation between the scattering dichroism and the thermodynamic component of the stress tensor (Bender and Wagner 1995). Figure 3 compares the $-45^{\circ}$ dichroism component with the total and thermodynamic component of the shear stress component for a suspension of $180 \mathrm{~nm}$ diameter silica spheres coated with 3(-trimethoxysilylpropyl)methylacrylate dispersed in tetrahydrofurfural (Bender and Wagner 1995). 
SD
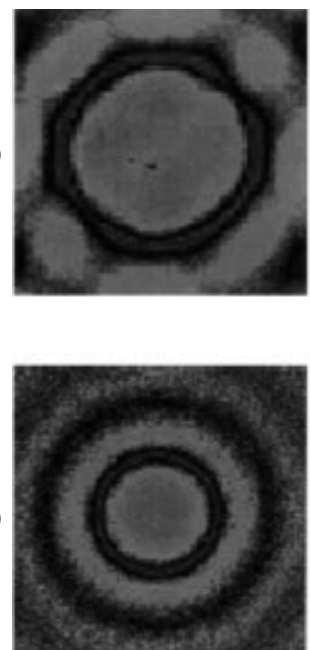

0.1
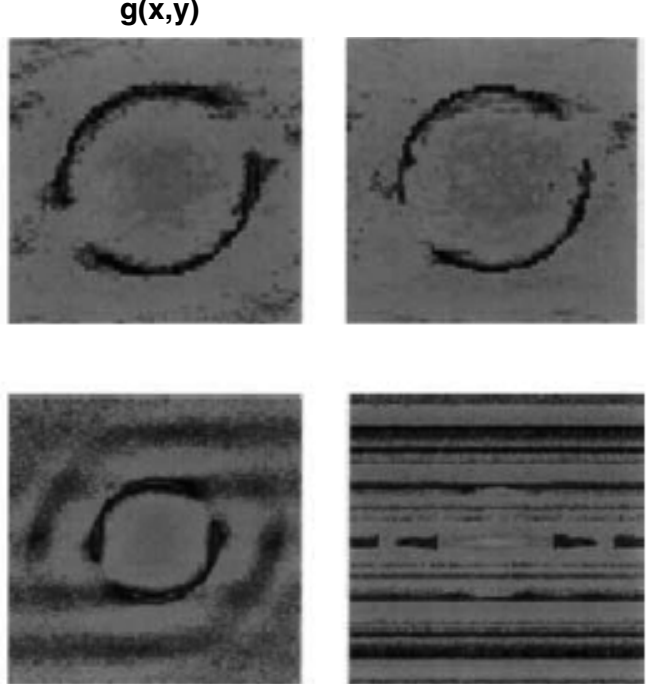

10

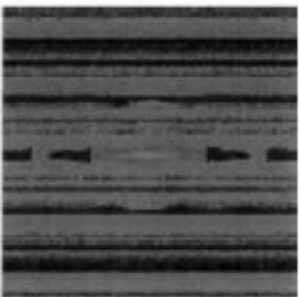

1000

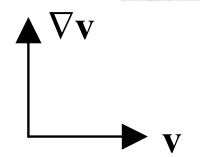

Figure 4. Suspension microstructure in the $\mathbf{v}-\nabla \mathbf{v}$ plane with (Stokesian Dynamic, SD) and without (Brownian Dynamics, BD) hydrodynamic interactions for three vales of the Pe number $(0.1,10$, 1000) (reprinted from Brady (2001), (C) (2001), with permission).

At high $P e$ numbers, the presence of shear thickening arises because of subtle changes in the distorted microstructure. Scattering dichroism and the colloidal stress-optical relation have been extremely useful in elucidating the microstructural origins of this transition. Shear thickening refers to an increase of the viscosity with increasing stress or Péclet number. Measurement of scattering dichroism (D'Haene et al 1993, Bender and Wagner 1996) and small angle neutron scattering (Maranzano and Wagner 2002) revealed cluster formation induced by hydrodynamic interactions. Maranzano and Wagner (2002) explicitly demonstrated the connection between the distorted microstructure and rheology with their 'stress-SANS' coefficient. More detailed information on the development of these hydroclusters was obtained from computer simulations (Brady and Bossis 1988, Brady 1996, Foss and Brady 2000, Catherall et al 2000, Brady 2001). In computer simulations of the shear-induced microstructure at high $P e$ numbers, for example in the shear thickening regime, the incorporation of hydrodynamic interactions is crucial. Figure 4 shows a comparison between simulations without hydrodynamics by the Brownian dynamics method (BD) and with hydrodynamics by Stokes dynamics (SD) for a hard-sphere suspension at $\Phi=0.45$ for Pe numbers equal to 0.1, 10 and 1000 (from Brady 2001). At the highest Péclet numbers the microstructures with and without hydrodynamics are radically different. With hydrodynamics, a distorted liquid-like structure is obtained. Without hydrodynamic interactions the particles arrange into strings along the flow direction, and the strings themselves are arranged in a hexagonal pattern. The BD method only predicts a shear thinning viscosity, whereas the SD method also accurately captures the shear thickening transition (Brady 2001). The transition arises as a consequence of the pronounced sharpening of the first nearest-neighbour peak in probability density along the compressive axes of the flow as is shown in figure 4 . This sharpening implies that particles are 
squeezed together until only a thin liquid layer separates them. This causes large hydrodynamic lubrication forces in the contact zone between particles. These forces actually diverge when the interparticle distance tends to zero; however, in experiments, short-range details such as surface roughness or stabilizing layer characteristics will intervene to modulate the divergence. The detailed physical mechanism of the shear thickening is somewhat subtler, and especially the role of Brownian motion as discussed by Bergenholtz et al (2002) for the case of binary collisions, but this discussion lies beyond the scope of the present review.

\subsection{Future prospects}

For the case of suspensions with an amorphous microstructure, the flow-induced changes in the microstructure are now well understood. A clear, even quantitative, link between the microstructure and non-equilibrium macroscopic properties has been established. Some challenges lie ahead in the high Péclet regime, where due to the extreme sensitivity of the lubrication forces to interparticle distance the details of interparticle potential (e.g. deformation of the polymer brush) or particle roughness on microstructure development need to be further understood (Mewis and Biebaut 2001, Wilson and Davis 2000, Melrose and Ball 2004).

\section{Effect of flow on the ordering of colloidal suspensions}

\subsection{Introduction}

Equilibrium colloidal suspensions adopt long-range order under certain conditions of interaction potential and volume fraction. Hoffman (1972) reported the first evidence that colloidal order can be manipulated by the application of shear flow. Other external fields will also shift ordering transitions (Hoffmann and Lowen 2001). In this section of the review, we first summarize the equilibrium phase behaviour of colloidal suspensions with hard-sphere and repulsive interactions. We then review the ordered structures that form upon application of flow. We describe how they are affected by shear rate and strain. Additional effects of colloidal volume fraction, interaction potential and particle polydispersity relevant to flowinduced ordering are identified. We examine molecular, Brownian dynamics and Stokesian dynamics simulations of order-disorder transitions in flow, and discuss the correspondence between simulation and experiment. Unresolved questions that warrant additional study are identified. Our discussion is restricted to suspensions of colloidal spheres.

As discussed in the introduction, the coupling between structural ordering and the nonequilibrium process of flow is of broad, fundamental interest in the fields of statistical mechanics and rheology. Suspensions of colloidal particles are a good model system for these studies because their interparticle structure occurs on scales that may be interrogated by light, x-ray and neutron scattering. Direct visualization techniques such as optical and confocal microscopy may also be applied. In addition to this fundamental science motivation, the application of flow to assist colloidal crystallization also has technological implications. For example, the development of large, defect-free ordered colloidal structures is a first step in bottom-up methods for assembly of materials such as synthetic opals for photonic band gap applications. Recent reports have applied shear flow in pursuit of this aim (Amos et al 2000, Sawada et al 2001). Fundamental studies of shear-induced ordering can inform the selection of the velocity profile. In addition, practical understanding of the behaviour in these simple flows can be used to engineer ordering in the more complex processes (such as evaporation (Jiang et al 1999)) that are of direct interest to the assembly of photonic band gap materials. 


\subsection{Phase transitions and equilibrium structure}

Suspensions of Brownian hard spheres undergo an equilibrium liquid-solid transition governed by the colloid volume fraction, $\phi$. The lowest free energy state is face centred cubic (fcc) (Woodcock 1997, Bolhuis et al 1997) and the coexistence region exists for volume fractions $0.494<\phi<0.545$. Poly(methyl methacrylate) colloids with polyhydroxystearic acid stabilizing chains dispersed in refractive index matching solvents are thought to closely approximate the hard-sphere interaction, although reports of subtle charge effects have recently appeared (Yethiraj and van Blaaderen 2003). These colloids have consequently been widely used as model systems. Scattering and visualization experiments with such materials have identified a coexistence region in good agreement with the predicted range (Pusey and van Megen 1986); however, the observed structure consists of close-packed layers with stacking registry that on average is intermediate between the fcc and hexagonal close-packed (hcp) structures (Pusey et al 1989, Zhu et al 1997). Charge interactions (Yethiraj and van Blaaderen 2003), sedimentation (Zhu et al 1997), finite size effects (Pronk and Frenkel 1999) and polydispersity (Auer and Frenkel 2001) may affect the degree of layer registry observed in the experimental systems that model the crystallization of hard spheres at equilibrium. In addition to these nonidealities, colloidal crystals often contain defects and grain boundaries. Co-existence of stacking faults and fcc twins has been observed (Kegel and Dhont 2000, Elliot et al 1997, Hoogenboom et al 2002). Finally, the growth of crystallites of hard spheres is retarded above the glass transition volume fraction $(\phi \sim 0.58)$.

Further experiments have quantified the homogeneous nucleation kinetics and structure of hard-sphere crystallization (Harland and van Megen 1997, Gasser et al 2001). Recent simulations have suggested that hard-sphere crystallization may occur by heterogeneous nucleation of a close-packed layer at a plane wall in preference to homogeneous nucleation (Auer and Frenkel 2003). The nucleated close-packed layer is then available to template bulk crystallization (Heni and Löwen 2000, van Blaaderen et al 1997).

Charged colloids that interact through screened Coulombic forces also crystallize; however, because of the repulsive Coulombic interaction, the transition to a colloidal crystal can occur even at dilute volume fractions if the screening (Debye) length is sufficiently large relative to the colloid size. Theory (Kremer et al 1986) and experiment (Sirota et al 1989) show that the phase diagram comprises both body centred cubic (bcc) and fcc structures. The bcc structure is found at low volume fraction and ion strength.

Polydispersity effects on crystallization structure and kinetics are difficult to quantify experimentally. Suspensions of polydisperse hard spheres are predicted to crystallize if the standard deviation in the size distribution (relative to colloid size) is below a maximum of 10\% (Arora and Tata 1998). Experimentally, the constraint on polydispersity might be even more severe since the crystallization kinetics retard dramatically as this theoretical limit is approached (Auer and Frenkel 2001). As opposed to atomic systems, nominally monodisperse colloid samples synthesized through special methods still commonly have relative standard deviations in size of a few per cent.

\subsection{Shear-induced structure in suspensions with hard-sphere and repulsive interactions}

Since Hoffman's (1972) pioneering work, the relationship among flow, ordered structures and shear stress has received extensive attention. Experimentally, light, neutron and x-ray scattering has been applied to measure the Bragg structure in a range of flow geometries and conditions. Interparticle interactions and colloid volume fractions have been systematically manipulated. The scattering of hypothetical real-space structures has been computed and 

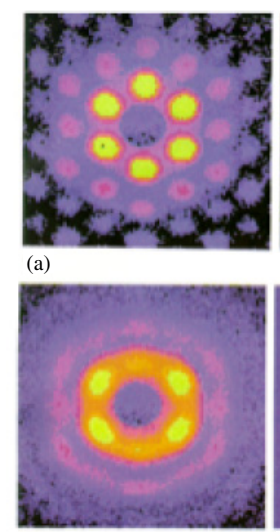

(d)

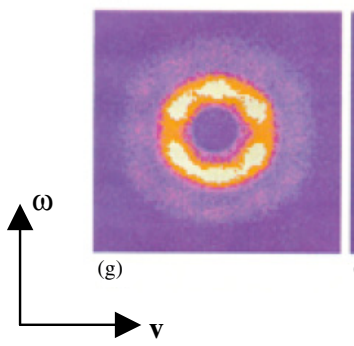

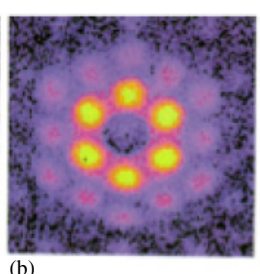

(b)

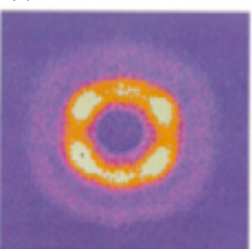

(e)

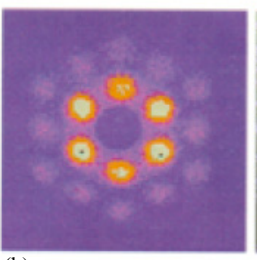

(h)

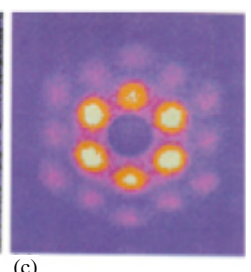

(c)

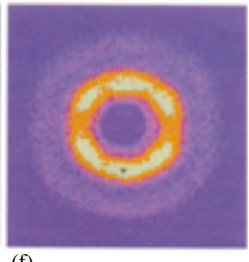

(f)

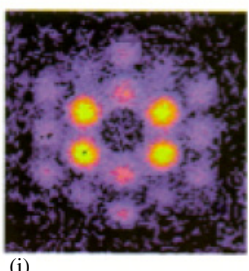

Figure 5. SANS scattering observed for a charge-stabilized suspension $(\phi=0.33)$ in the configuration of figure 1(b) (v- $\omega$-plane). The shear rates at which these steadystate structures were observed are: (a) at rest; (b) $0.008 \mathrm{~s}^{-1}$; (c) $0.02 \mathrm{~s}^{-1}$; (d) $0.15 \mathrm{~s}^{-1}$; (e) $0.3 \mathrm{~s}^{-1}$; (f) $1.5 \mathrm{~s}^{-1}$; (g) $15 \mathrm{~s}^{-1}$; (h) $100 \mathrm{~s}^{-1}$; (i) $11000 \mathrm{~s}^{-1}$ (reprinted from Chen et al $(1994 \mathrm{a}$, 1994b), (C) (1994), with permission).

compared to experiments. More recently, computer simulation and microscopic visualization have become complementary methods for structural investigations.

Most experiments have been conducted in either steady-shear or oscillatory-shear flow. Small-gap Taylor-Couette, capillary slit and parallel plates geometries have been common geometries. In simple shear flow, the deformation is specified by the shear rate, $\dot{\gamma}$, and the strain, $\gamma$. We divide our treatment into a discussion of structures in steady-shear and oscillatory-shear flow.

3.3.1. Effect of the steady-shear rate on ordered colloidal structures. The structure of chargestabilized latex suspensions subjected to steady-shear flow has been investigated by small-angle neutron (SANS) and synchrotron x-ray scattering. The steady-shear flow has typically been generated in rotational Couette flow or parallel plate flow. The correspondence between the observed structural states and shear-rate dependent rheology has also been investigated.

With the incident radiation propagating along the centreline of the shear cell (figure 1(b)), for a system with only two-dimensional order, the diffraction patterns in the $\mathbf{v}-\omega$ plane would persist as tubes of intensity upon shifting $\mathbf{q}$ into the gradient direction $\left(q_{\nabla} \neq 0\right)$ (Guinier 1963, Loose and Ackerson 1994). Three-dimensional order leads to intensity modulation in the gradient direction, and perfect three-dimensional order causes the tubes of intensity to break up into nodes in three-dimensional q-space. Measurements of scattering intensity at non-zero $q_{\nabla}$ are accomplished, for example, by shifting the shear cell relative to the incident source, as was shown in figure 1(c). In other flows, such as parallel plate flows, rotation of the flow about an axis accomplishes a similar translation in $q_{\nabla}$ (Dux et al 1998, Versmold et al 2001). Analysis of scattering data for close-packed planes with layer ordering may be accomplished by methods described in Loose and Ackerson (1994) and Guinier (1963).

To qualitatively demonstrate the effect of shear rate, SANS data (from Chen et al 1994a) collected in the figure 1(a) configuration, are reproduced in figure 5. The volume fraction of 


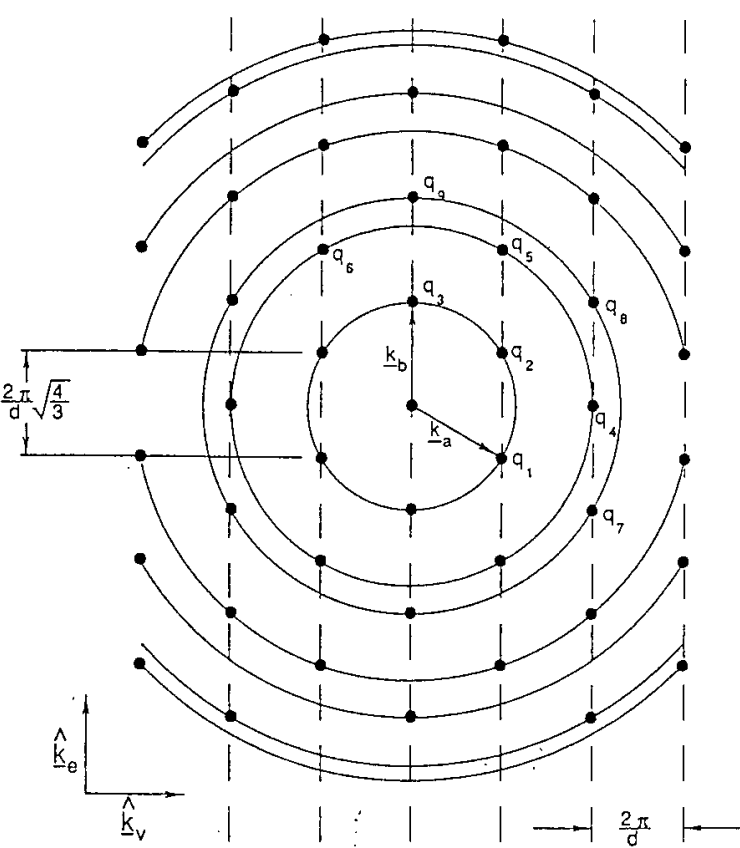

Figure 6. Diffraction pattern for an ideal 2D hcp structure. The lattice basis vectors $\mathbf{k}_{a}$ and $\mathbf{k}_{b}$ are shown (reprinted from Loose and Ackerson (1994), (C) (1994) with permission).

this charge-stabilized colloidal suspension is $\phi=0.33$. The rest structure, shown in figure 5(a), is consistent with order with hexagonal structure in the plane parallel to the shearing surface. The close-packed layers have registry intermediate between hcp and fcc. That is, the layer registry includes examples of the fcc twin structures (ABCA, ACBA) and the hep structure $(\mathrm{ABAB})$. Here the alphabetic indexing notation identifies the stacking of close-packed layers.

As the shear rate is increased, the charge-stabilized suspension shows three regimes of behaviour. At low shear rates $\left(\dot{\gamma} \leqslant 0.02 \mathrm{~s}^{-1}\right)$, the six-fold symmetry of the diffraction pattering persists but the intensities of the two inner nodes on the vorticity axis reportedly decrease relative to the quiescent measurement. For $0.15 \mathrm{~s}^{-1} \leqslant \dot{\gamma} \leqslant 15 \mathrm{~s}^{-1}$, scattering in the flow-vorticity plane appears as concentric rings of intensity (with some azimuthal modulation in intensity). The rings are reminiscent of a powder diffraction pattern. Inner diffraction nodes with six-fold symmetry reappear for measurements at $\dot{\gamma}=100 \mathrm{~s}^{-1}$ and persist to the highest shear rate studied $\left(11000 \mathrm{~s}^{-1}\right)$.

The observations of six-fold symmetry in the scattering patterns for $q_{\nabla}=0$ are consistent with close-packed structure in the flow-vorticity plane. Additional information about ordering along the gradient direction is available from diffraction measurements at $q_{\nabla} \neq 0$ (accessed by means of the configuration of figure 1(b) for example) (Ackerson et al 1986, Clarke et al 1997, Dux et al 1998, Versmold et al 2001). If the registry of the close-packed layers is random, then the nodes observed at $q_{\nabla}=0$ will extend as tubes of uniform scattering intensity in a direction parallel to the gradient axis. Ordering in the gradient direction causes modulation of intensity along the tubes. Here we limit our discussion to the inner diffraction nodes because form factor effects often attenuate the higher-order reflections.

For diffraction from close-packed layers with incomplete registry, the scattering vector, q, may be conveniently described as

$$
\mathbf{q}=h \mathbf{q}_{a}+k \mathbf{q}_{b}+l \mathbf{q}_{c} .
$$

Here, as shown in figure 6 (Loose and Ackerson 1994), the basis vectors $\mathbf{q}_{a}$ and $\mathbf{q}_{b}$ describe a $2 \mathrm{D}$ close-packed structure oriented perpendicular to the gradient direction. $\mathbf{q}_{c}$ is oriented along 


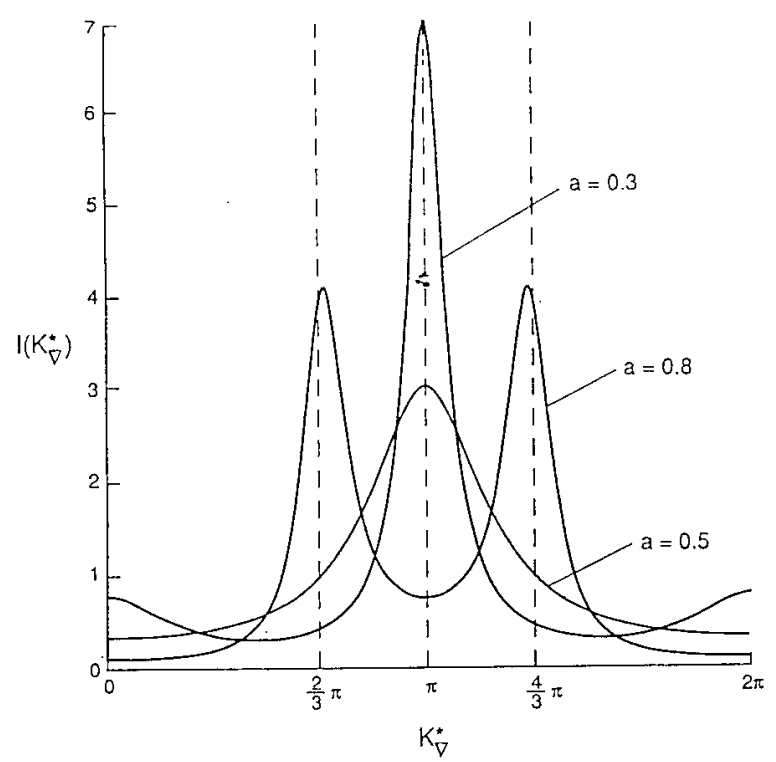

Figure 7. Variation of $I(l)$ at a diffraction node of the inner ring of a $2 \mathrm{D}$ hcp structure for various values of the stacking probability, a. The curves quantify the modulation in diffraction intensity along tubes parallel to the gradient direction (reprinted from Loose and Ackerson (1994), () (1994) with permission).

the gradient direction with characteristic magnitude $d \sqrt{2 / 3}$, the distance between close-packed fcc layers. Here $d$ is the nearest-neighbour distance. The diffraction intensity is non-zero for integer values of the index $(h, k)$. If the close-packed layers are not in perfect registry, the diffraction intensity, $I(l)$ varies continuously in the gradient direction.

$I(l)$ differs for nodes with indices that satisfy either $h+k=3 n$ or $3 n \pm 1$, where $n$ an integer. The stacking probability, $a$, specifies the propensity for fcc ( $a=1$ is fcc) versus hcp ( $a=0$ is hcp) order of the close-packed layers. $a=1 / 2$ is the special case of random registered stacking. Figure 7 (Loose and Ackerson 1994) shows the theoretical effect of $a$ on $I(l)$ for a tube with $h+k=3 n \pm 1$. Figure 8 shows analogous measurements by Dux et al (1998) for a charge-stabilized suspension after shearing at $\phi=0.13,0.18$ and 0.31 . Comparisons between theory and experiment for the $h+k=3 n$ rods are also available (Dux et al 1998, Chen et al 1994a, Ackerson et al 1986, Versmold et al 2001).

Hypothetical real-space structures have been evaluated against both measurements of the diffraction intensity at $q_{\nabla}=0$ (such as figure 5) and the intensity modulation along particular tubes in the gradient direction (such as figure 8). Two such candidate real-space structures, after Loose and Ackerson (1994), are shown in figure 9. In figure 9(a), relative deformation of layers is accomplished by the mechanism of a strained crystal. In figure 9(b), motion by sliding layers is shown. The low-shear rate scattering regime is consistent with the strained crystal structure (e.g. figures 5(b) and (c)). At high shear rates the data are well described by the sliding layer mechanism (e.g. figures 5(h) and (i)). These structures are closely related to trajectory paths that accommodate the imposed strain field with the minimal energetic penalty and structural distortion.

The behaviour at intermediate shear rates (e.g. figures $5(\mathrm{~d})-(\mathrm{g})$ ), where the diffraction resembles a powder pattern, has been alternatively described as consistent with polycrystallinity (Chen et al 1994a) or orientationally disordered layers (Versmold et al 2001). Small ordered regions have been observed in this regime by photographic visualization of the bulk flow (Chen et al 1992, 1994b). This regime is also associated with discontinuous and hysteretic rheology (Chen et al 1992, 1994a). A recent direct visualization study of rotating domains in a 2D colloidal crystal sheds light on one possible mechanism of behaviour in this regime (Stancik 
(a)

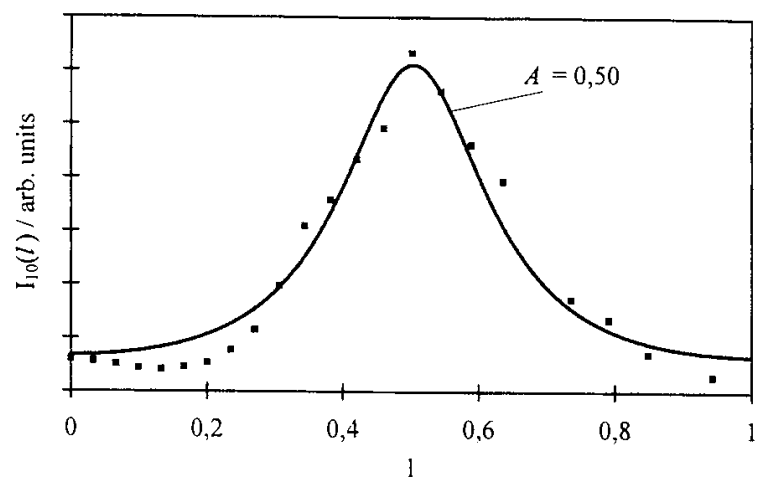

(b)

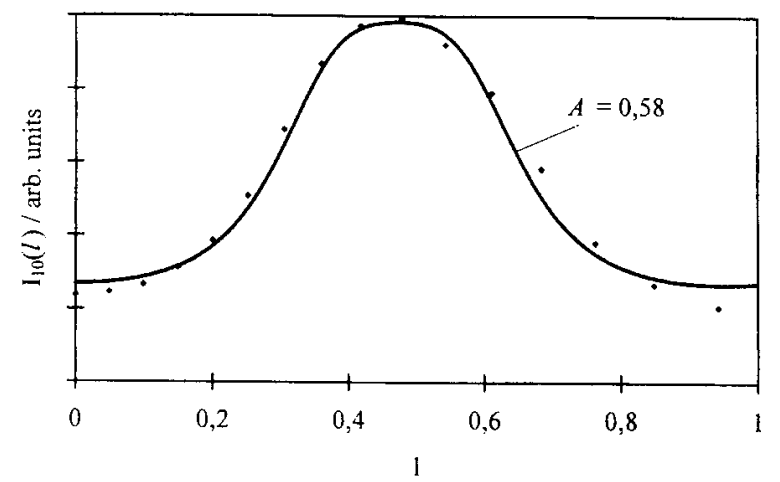

Figure 8. Experimental measurements of the variation in tube intensity parallel to the gradient direction for conditions analogous to figure 5 or sheared charge-stabilized suspensions of volume fraction $\boldsymbol{\square}=0.13,0.18$ and 0.31. The curves are fits of the stacking parameter $a$. The structures are intermediate between random layer registry $(a=0.5)$ and fcc order $(a=$ 1.0) (reprinted from Dux et al (1998), (C) (1998) with permission).

et al 2004). Stokesian dynamics simulation of non-Brownian, electrostatically stabilized particles has characterized the pathways that particles track as close-packed layers undergo relative translation due to shear flow. The sequence of trajectories evolves from the zigzag motion characteristic of the strained crystal to the rectilinear motion of the sliding layer mechanism. Interestingly, when the crystal orientation is constrained so that the [211] direction of a (111) close-packed plane is oriented in the flow direction, large jumps in viscosity and hysteresis occur in constant shear rate Stokesian dynamics simulations (Gray and Bonnecaze 1998). (Note that the [211] direction is perpendicular to the close-packed direction of the (111) plane.) Differences between the Stokesian dynamics study and earlier simulations (Stevens and Robbins 1993) that did not incorporate hydrodynamic interactions suggest that the discontinuous behaviour is a sensitive function of the balance between electrostatic forces and hydrodynamic interactions. 

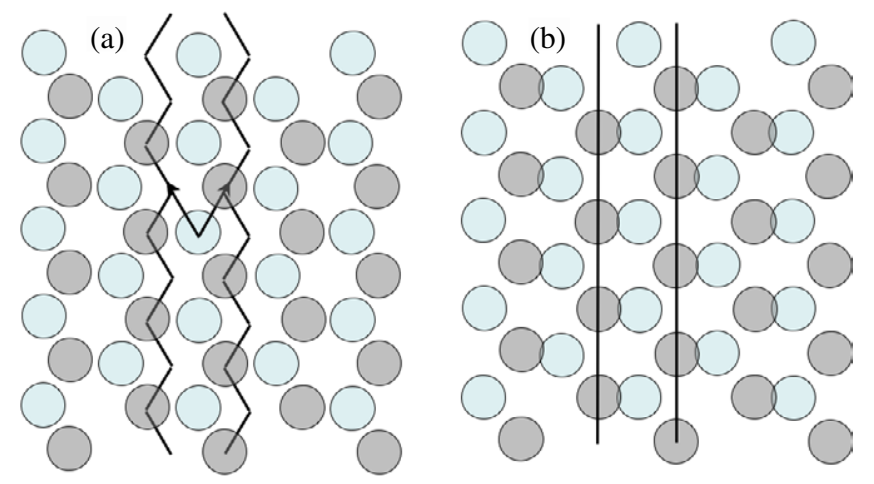

Figure 9. Proposed mechanisms for relative displacement of close-packed layers oriented normal to the gradient direction. A strained crystal in which layers undergo zig-zag motion is shown in (a). In (b), the sliding layer configuration is shown. The flow direction is horizontal. After Loose and Ackerson (1994).

The sequence of transitions described above is neither complete nor universal. For example, the intermediate, orientationally disordered, regime is not reported in all cases. Moreover, an initially crystalline colloidal suspension with weakly repulsive interactions exhibited a regime of one-dimensional string-like motion (strings oriented in the flow direction in real space) at high shear rates (Yan and Dhont 1993). Bundle ordering (Vermant et al 1999) (in the flow direction) is also found at high shear rates. As the shear rate is further increased, shear melting occurs (Ackerson and Clark 1981, Ashdown et al 1990, Yan and Dhont 1993). Finally, also at high shear rates, a nearly disordered charge-stabilized suspension has been observed to relax upon flow cessation into a metastable configuration of randomly stacked hcp planes (Butera et al 1996).

Modern non-equilibrium molecular and Brownian dynamics simulations have been applied to evaluate the conditions necessary to induce ordered structures in shear flow. Such work is also critical because it sheds light on the mechanistic origins. In addition, simulations can efficiently study the dependence of a transition critical stress (or Péclet number) on material parameters such as volume fraction and degree of repulsive interaction. Mitchell et al (1995) performed Brownian dynamics simulations of near hard spheres with initially disordered structure subjected to steady-shear flow. A Péclet number dependent transition to an ordered structure of particle strings aligned in the flow direction was observed. At high Péclet number the strings further organize in a hexagonal structure. This transition was observed at volume fractions as low as $\phi=0.31$. Simulations of charge-stabilized colloids $(\phi=0.267)$ in large systems $(N=43000)$ also show string formation (Rastogi et al 1996a). In this case, the hexagonal ordering observed at $P e=15$ is already quite complete. Brownian dynamics simulations of this kind neglect the coupling between hydrodynamic interactions and structure that can become important at high volume fraction and Péclet number (see section 2.2). However, flow-aligned strings with hexagonal organization in the gradientvorticity plane are still observed above the equilibrium hard-sphere coexistence boundary when hydrodynamic interactions are included (Sierou and Brady 2002). This observation was made by means of accelerated Stokesian dynamics simulation for the particular case of non-Brownian suspensions.

The aforementioned discussion concerned close-packed structures. At low volume fraction and ionic strengths where the equilibrium structure is bcc, Ackerson and Clark (1984) observed shear-induced structural modifications. In this case, shear flow orients the bcc crystal 
(a)

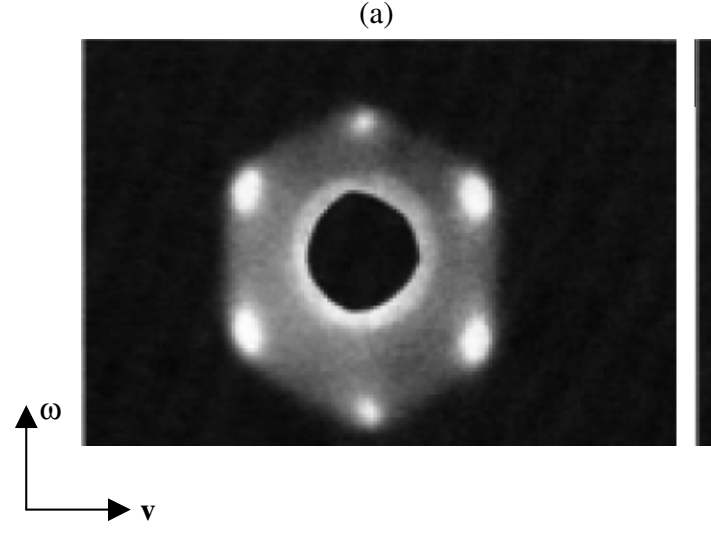

(b)

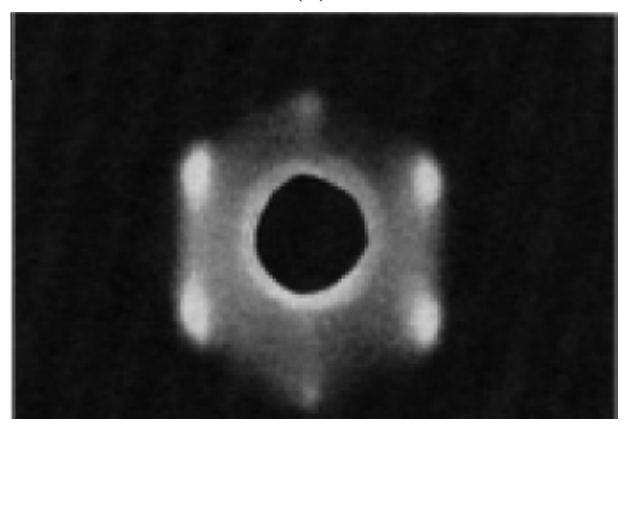

Figure 10. Three-fold inner ring diffraction pattern $\left(q_{\nabla}=0\right.$, plane: $\left.\mathbf{v}-\omega\right)$ for a $\phi=0.485$ suspension of near hard spheres observed during oscillatory shear. The two patterns are consistent with fcc twins. The structure alternates between twin structures during each cycle of the oscillation (reprinted from Ackerson (1990), ( (1990) with permission).

so that a (110) plane is perpendicular to the gradient direction. A [111] direction of the unit cell is aligned in the flow direction. This configuration admits twin structures. As the shear rate is increased, the bcc structure in the (110) plane is progressively strained to resemble a distorted close-packed hcp structure. At higher shear rates the distorted 2D hcp planes slide relative to each other as discussed above. In the non-equilibrium molecular Brownian dynamics simulation, the observed orientation at low shear rates is found to persist after the application of flow. Other initial configurations are destroyed by the shearing action (Butler and Harrowell 1995).

For charge-stabilized suspensions, steady-shear flow may induce layer order in suspensions with liquid structure at rest (Ashdown et al 1990, Laun et al 1992). Such fieldassisted self-assembly is of technological interest (van Blaaderen 2004). The measurements are also relevant to the theory of non-equilibrium phase transitions (Butler and Harrowell 2002). However, for suspensions with hard-sphere-like interactions at volume fractions below the coexistence region, only string-like (Ackerson 1990) order has been observed. In contrast to simulation results, there have been no experimental reports of close-packed layer ordering or string organization below the coexistence volume fraction for hard spheres; however, in the two-phase region layer ordering has been reported (Ackerson 1990).

Most shear-induced ordering experiments have been conducted with nearly monodisperse suspensions (standard deviation in particle size $<5 \%$ of the mean is typical). Such tight constraints on size distribution are consistent with end-use applications involving self-assembly. Earlier we noted that, at equilibrium, polydispersity dramatically affects the crystallization boundaries and kinetics. Brownian dynamics simulations suggest that polydispersity similarly inhibits the production of ordered phases in steady-shear flow (Rastogi et al 1996b).

3.3.2. Structural ordering induced by oscillatory-shear flow. Early oscillatory-shear studies demonstrated the remarkable fact that crystal order can be induced in suspensions of spheres with near hard-sphere interactions even when the equilibrium structure is liquid-like (Ackerson and Pusey 1988). The model system consisted of sterically stabilized poly(methyl methacrylate) spheres suspended in approximately refractive index and density matched solvents. Figure 10 reports, from Ackerson (1990), two flow-vorticity plane $\left(q_{\nabla}=0\right)$ 
diffraction patterns (oscillatory frequency $=3.3 \mathrm{~Hz}$ and $\phi=0.485$ ). The two patterns were acquired, approximately, at the minimum and maximum displacement of the oscillation. Each three-fold pattern is consistent with a single twin fcc structure with a close-packed (111) plane oriented parallel to the shearing surface. Physically, the three peaks correspond to diffraction from the other three sets of (111) planes of the fcc crystal. Interestingly, here the close-packed direction in the (111) plane is oriented in the vorticity direction (perpendicular to its orientation in steady-shear flow) (Ackerson and Pusey 1988, Ackerson 1990, Yan and Dhont 1993). Direct visualization studies (Haw et al 1998) have suggested that a distribution of orientations of fcc crystal grains about the vorticity direction accounts for the observed diffuse intensity (see figure 5) in the azimuthal direction about the expected fcc diffraction peak.

Ackerson and Pusey (1988) hypothesized that the observed orientation of the closepacked direction facilitates easy translation between the two fcc twin structures at strains of approximately one. (Note that here $\gamma$ is defined on a peak to peak basis, consistent with the literature on this topic; thus, strain amplitudes quoted are twice those based on the usual definition in rheology.) At higher strain amplitudes, the fcc structure persists but with the close-packed direction now oriented in the flow direction (Ackerson 1990). The sequence of structural transitions as the strain amplitude is stepped up is rich. Observations have been summarized by Ackerson (1990) at a constant deformation rate $(P e>24)$ for a hard-sphere suspension with liquid-like order at equilibrium. Regimes of one-, two-and three-dimensional order are all observed, as well as the existence of hysteresis.

For experiments at $P e>1$ and $\gamma \sim 1$, it appears that structure is built up by successive interactions between neighbouring particles; steady-state crystallinity is achieved after hundreds to thousands of cycles. If the volume fraction is below coexistence, the ordered structure is metastable upon cessation of flow (Ackerson and Pusey 1988). For example, structural decay occurred after about $\sim 30 \mathrm{~min}$ for one study at $\phi \sim 0.48$. Suspensions with weak repulsive interactions order similarly when oscillatory shear is applied (Yan and Dhont 1993). The kinetics of order formation under oscillatory shear has recently been studied for an aqueous sterically stabilized suspension (Panine et al 2002).

\subsection{Unresolved issues and future prospects}

Our review has demonstrated that, since the appearance of the first report more than thirty years ago, remarkable progress has been made in characterizing the nature of flow-induced ordering in colloidal suspensions. The ordering is surprisingly widespread-it has been observed for a host of equilibrium structures over a broad range of deformation rates. The phenomenology is also almost bewilderingly rich - a complex sequence of morphological transitions governing the organization of close-packed layers in steady-shear and oscillatoryshear deformation have been described. Progress in evaluating the consistency of simple structural models with the intricate diffraction patterns observed by scattering may be judged to have been broadly successful. A next step, detailed comparison between experiment and direct simulation, is beginning to bear fruit. Trends toward simulation of large system sizes and inclusion of hydrodynamic interactions will certainly enrich the opportunity for deep mechanistic understanding of shear-induced ordering in systems with hard-sphere and repulsive interactions. Such work with model colloidal particle suspensions has implications for the behaviour of other types of complex fluids with particle-like constituents such as block copolymers (Eiser et al 2000).

It is clear that definitive identification of the shear-induced crystal structure from scattering requires experimental configurations in which the relative orientation of the scattering and flow coordinate frames may be manipulated. These configurations are challenging to realize. 
Moreover, form factor effects complicate the quantification of diffraction peak intensity and hinder resolution of higher-order reflections helpful for unambiguous indexing. In addition, some scattering studies have found mixed or coexisting morphologies, and the real-space structure leading to a particular scattering pattern has not been definitively resolved in all cases. Because they interrogate the time-averaged structure over a scattering volume that is macroscopically large, scattering measurements distinguish between spatial and temporal structural fluctuations only with difficulty. Inhomogeneous strain of the sample, especially at the shear flow boundaries, is also difficult to resolve by means of scattering. Finally, the dynamical role of microscopic vacancies, amorphous disorder and grain boundaries in generating morphology and mediating structural transitions can typically only be inferred from scattering studies.

Attention to these issues would facilitate further characterization of ordering mechanisms and more detailed comparison between experiment and simulation. Direct visualization studies by optical and confocal microscopy can contribute useful information about local structure and dynamics. For example, confocal microscopy has already resolved new aspects of crystallization in equilibrium and sedimenting systems (Yethiraj and van Blaaderen 2003, Hoogenboom et al 2002, Gasser et al 2001). 2D visualization of flow-induced structure has been accomplished by means of phase contrast microscopy (Haw et al 1998) or by visualizing 2D suspensions (Stancik et al 2003, 2004). Using the latter systems the effects of extensional flows on colloidal crystals have also been explored (Stancik et al 2002). Recent reports of the execution of confocal microscopy in conjunction with an applied flow (Varadan and Solomon 2003b, Biehl and Palberg 2004, van Blaaderen 2004) suggest that 3D direct visualization methods may be fruitfully applied to further advance the study of flow-induced order in colloidal suspensions.

\section{Gelling suspensions}

\subsection{Introduction and relevance}

Suspensions that undergo a fluid-to-gel transition are of enormous technological significance, partly because the gel microstructure and mechanical properties can be controlled. In addition, gelation is a common, but unwelcome, outcome of attempts at field-assisted colloidal assembly. In the absence of external fields, attractive colloidal particles at low volume fraction organize themselves as fractal aggregates. At sufficient volume fraction, the aggregates form a spacefilling gel. The resulting clusters or flocs possess a hierarchy of self-similar structures; over a wide range of length scales the microstructure can be characterized by a single fractal dimension $\left(n \sim r^{d_{\mathrm{f}}}\right)$ (Schaefer et al 1984). The fractal dimensions, $d_{\mathrm{f}}$, range from 1.75 for diffusion limited cluster aggregation (DLCA) to 2.1 for reaction limited cluster aggregation (RLCA) (Bushell et al 2002). Fractal dimensions can fall outside this range when internal restructuring is possible due, for example, to Brownian motion or flow. Typical values for thermoreversible aggregation are close to 2.4 (Rueb and Zukoski 1997, Varadan and Solomon 2001). Thermoreversible gelation of adhesive spheres is thought to involve the development of a short-range attractive interaction between particles due to temperature-induced changes in steric layer conformation at the surface of adhesive spheres (Grant and Russel 1993).

As the colloidal volume fraction is increased above about $10 \%$, structural models based on fractal self-similarity increasingly can fail because the tenuous open fractal structure is inconsistent with a high particle loading. At high particle loading, reversible gelation instead leads to local voids and long length scale fluctuations in particle number density (Varadan and Solomon 2003a, Shah et al 2003). Irreversible, reaction limited colloidal aggregation 
in concentrated dispersions on the other hand leads to dense, fractal structures around the gel point that can be detected quantitatively by both rheological and dynamic light scattering methods (Elliott et al 2003).

The fractal-cluster structure also controls the linear viscoelastic rheological properties of aggregated dispersions (Shih et al 1990, Trappe and Weitz 2000). A low-frequency plateau of the storage modulus occurs and the effects of particle size and colloidal interactions have been elucidated (Buscall et al 1987, 1988, Sonntag and Russel 1987, Chen and Russel 1991, Vanderaerschot and Mewis 1992, Rueb and Zukoski 1997). Some of the nonlinear rheological properties, such as the yield stress, correlate well with the evolution of the linear viscoelastic ones (Shih et al 1990, Rueb and Zukoski 1997). Recent scaling arguments suggest universality in terms of a jamming phase diagram, unifying the behaviour of many systems as a function of the particle volume fraction, the energy of interparticle attractions, and the applied stress (Prasad et al 2003). Whereas some success has been achieved using a microstructural approach based on fractal concepts to predict the zero-shear viscosity (Potanin et al 1995), it cannot model the strong shear-rate dependence of the viscosity (Woutersen and Dekruif 1991, Vanderaerschot and Mewis 1992) or the general features of the thixotropy (time dependence) (Mewis 1979, Barnes 1997). For systems at low volume fractions, containing individual aggregates, simulations by Doi and Chen have elucidated the dependence of viscosity on aggregate geometry (Chen and Doi 1999). As in sections 2 and 3, Stokesian and Brownian dynamic simulations have been used as an approach for more concentrated suspensions. Brownian dynamics simulations were used to assess the linear viscoelastic rheological properties of the particulate network (Dickinson 2000). Stokesian dynamics simulations for attractive colloids suggest that the nonlinear rheological properties are dominated by stress-bearing structures building up during the flow (Silbert and Melrose 1999, Silbert et al 1999b, 1999a). The understanding of the flow-induced structures is, however, not yet as complete compared to the stable suspensions (sections 2 and 3). Therefore, in the next section, we will review the experimental work on the flow-induced changes in sheared aggregated colloidal dispersions in more detail.

\subsection{Flow-induced structure in aggregated systems}

Flow will affect the size, density and structural organization of the flocs. Vandeven and Mason (1977a, 1977b) were the first to systematically study the effects of flow on the distribution of the particles over singlets, doublets, and triplets (and higher) at the entrance and the exit of a capillary tube, for different shear rates in the high Péclet number regime. In addition to changes in size, flow will also lead to a densification of individual aggregates (Torres et al 1991, Hoekstra et al 2003). Since the aggregate size is set by a competition between the attractive colloidal forces and the disrupting hydrodynamic forces, it has been suggested that a fractal aggregate concept can be used to explain the observed tendencies (Tolpekin et al 2004). The situation becomes more complex as the volume fraction is increased. Size reduction and flow densification are still observed (Rueb and Zukoski 1997, Varadan and Solomon 2001, Hoekstra et al 2003), but the self-similarity of the structure breaks down during shear flow as the microstructure becomes anisotropic. Small-angle light scattering experiments on aggregated suspensions using a set-up as in figure 1(b) reveal a characteristic two-lobe 'butterfly' scattering pattern (DeGroot et al 1994, Verduin et al 1996, Pignon et al 1997, Varadan and Solomon 2001, Vermant 2001, Hoekstra et al 2003), an example of which is shown in figure 11. The long axis of the scattering pattern is oriented in the flow (v) direction.

Significantly, butterfly scattering patterns are quite common in sheared complex fluids. Bastide et al (1990) were the first to observe them using SANS from deformed (stretched) swollen gels. In the case of deformed gels, the patterns arise as a consequence of a flow- 


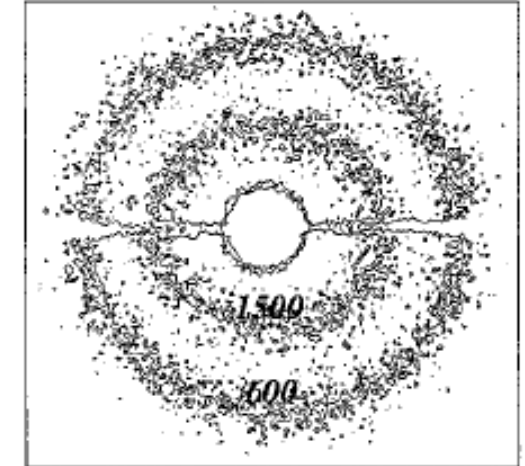

$\dot{\gamma}=1.2 \mathrm{~s}^{-1}$

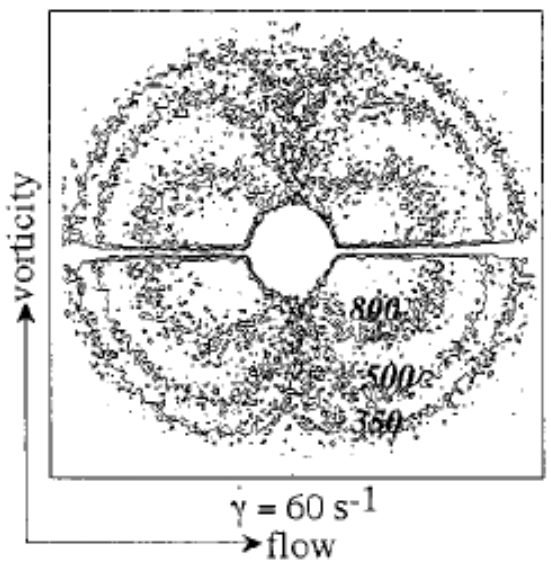

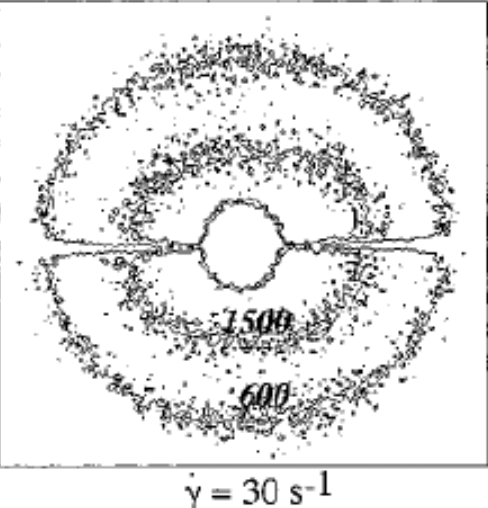

$\dot{y}=30 \mathrm{~s}^{-1}$

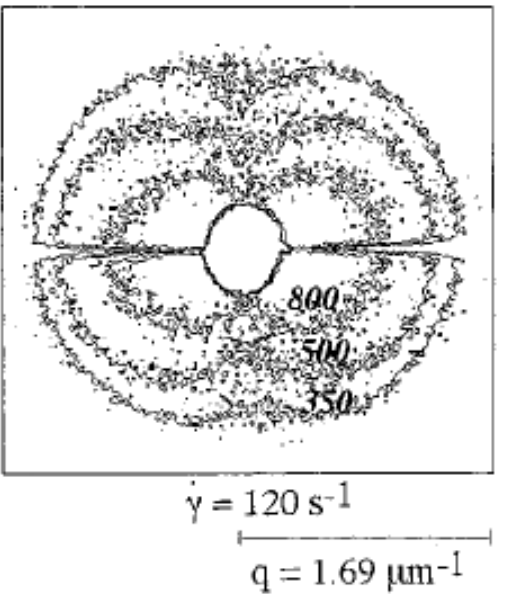

Figure 11. Isointensity contours showing the evolution of the anisotropy in the $\mathbf{v}-\omega$ plane at high shear rates for organophilic silica-hexadecane gels at $\phi=0.1$. The line crossing the images is due to a wire that contains the beamstop (reprinted with permission from Varadan and Solomon (2001), (C) (2001) American Chemical Society).

enhancement of local scale in homogeneities. Apart from the weakly aggregated sticky sphere dispersions, the patterns are displayed in SALS experiments from a variety of complex fluids subjected to shear flow. Examples include semi-dilute polymer solutions (Hashimoto and Kume 1992, Van Egmond et al 1992, Moses et al 1994, Boue and Lindner 1994, Van Egmond 1997), polymer blends with a viscoelastic asymmetry (Hobbie and Migler 1999, Hobbie et al 2002), polymer microgels (Stieger and Richtering 2003), micellar wormlike surfactants (Wheeler et al 1996, Kadoma and van Egmond 1997, Schubert et al 2004), different polymer-colloid mixtures such as nanocomposites (Lin-Gibson et al 2004) and mixtures of associative polymers and latex particles (Belzung et al 2000). In the latter case, no evidence of particle aggregation was observed in the rheological behaviour. In the case of associative polymer/particle mixtures and for semi-dilute polymer solutions, a clear maximum in the scattering curves is present, which evolves from large to small $\mathbf{q}$ as time evolves. This maximum and the corresponding time evolution are not observed for suspensions of weakly aggregated suspensions. For viscoelastic polymer solutions, scattering anisotropy of the kind described above has been understood to be the consequence of shear-induced enhancement of concentration fluctuations (Helfland and Fredrickson 1989, Fuller 1995). 

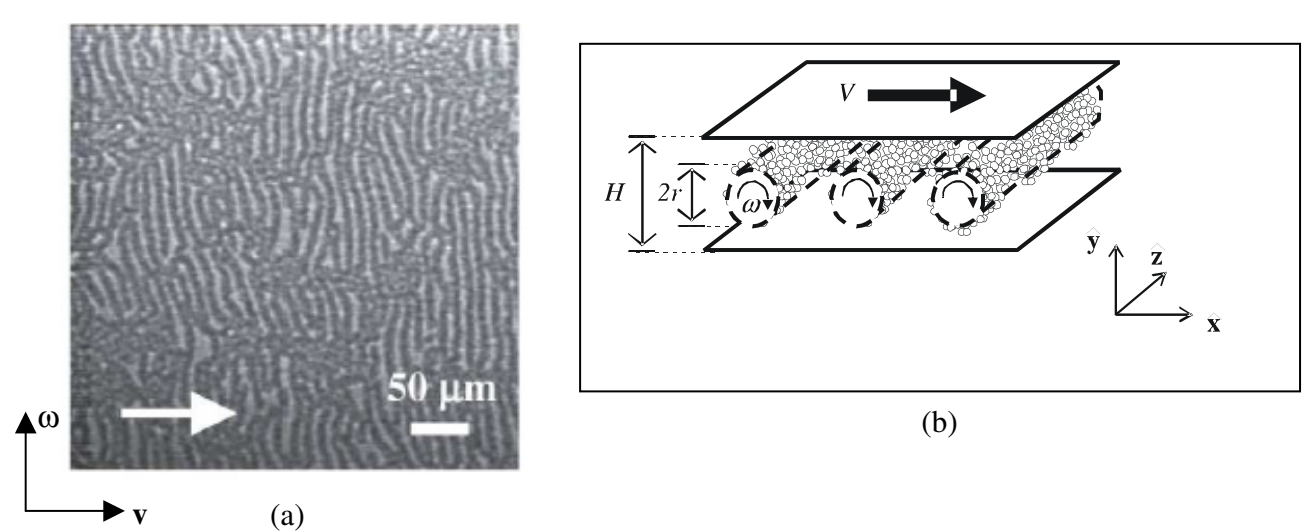

(b)

Figure 12. (a) Microphotographs of a depletion flocculated water-oil emulsion $(\phi=0: 58)$ stabilized under simple shear for gap thickness $12 \mu \mathrm{m}$. The plane of observation is the $\mathbf{v}-\omega$ plane. (b) Schematic representation of the proposed roll-cell mechanism and conformation of cylindrical flocs (reprinted with permission of Montesi et al (2004), () (2004) by the American Physical Society).

The microscopic picture underlying the butterfly scattering pattern in flowing suspensions has been discussed by DeGroot et al (1994), who compared their SALS observations on agglomerated silica particles in PDMS liquids with video microscopy showing the presence of roller-like structures. They hypothesized that aggregates wrap around themselves. This organization causes them to contract in the flow direction and extend along the vorticity axis. This structure is consistent with enhanced concentration fluctuations in the flow direction and thus the observed butterfly scattering. Similar roller-like structures have been observed by microscopy in weakly attractive emulsions as is shown in figure 12 (Montesi et al 2004) and in flows of nanotube suspensions in confined geometries (Lin-Gibson et al 2004). For the latter two systems it has been suggested that cylindrical flocs form, align along the vorticity axis, and undergo a log-rolling movement. It is thought that this structure is associated with large negative first normal stress differences; however, for the case of aggregated suspensions this implication of the structure has not been reported.

The butterfly light scattering patterns observed in shear flow of aggregated colloidal dispersions are indicative of structure anisotropy on dimensions much larger than the primary particle size. There is some experimental evidence that shear can also render the local microstructure anisotropic. For example, neutron scattering patterns of sheared sticky (adhesive) sphere dispersions were anisotropic at high shear rates; an example is shown in figure 13 (Woutersen et al 1993). Neutron scattering experiments on the thixotropic clay dispersions on the other hand displayed no small length scale anisotropy (Pignon et al 1997).

The number of simulation studies on aggregated colloidal dispersions in shear flow is limited. Calculated structure factors for flowing concentrated attractive spheres projected in the velocity-vorticity plane reveal a distortion at large values of the scattering vector (Silbert and Melrose 1999). In this case the anisotropy is related to string formation of particles. This simulation implies that on local length scales (comparable to the particle size) anisotropic particle organization can be obtained in these systems, as previously observed by Woutersen et al (1993). Comparing the experiments (figure 13) and the simulations, however, reveals important differences. Whereas the SANS results show a two-lobe pattern comparable with light scattering results on aggregated suspensions, the simulations predict arrays of Bragg-like peaks, oriented in the vorticity direction, slicing through an otherwise liquid-like structure factor. 


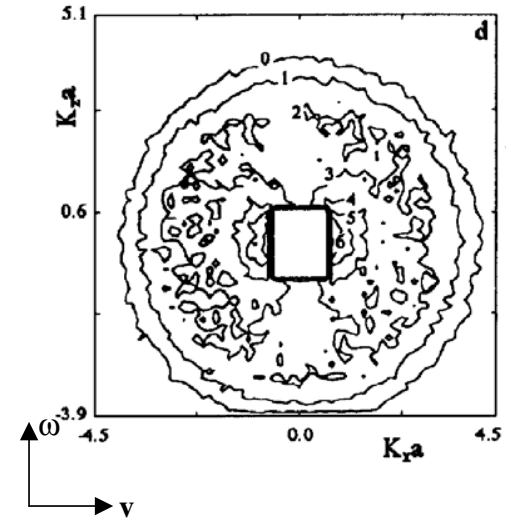

Figure 13. (a) Neutron scattering pattern at high shear rate for a sticky sphere dispersion of silica particles in benzene at a volume fraction of 0.38 in the $\mathbf{v}-\omega$ plane (reprinted with permission from Woutersen et al (1993), (C) (1993) the Society of Rheology).

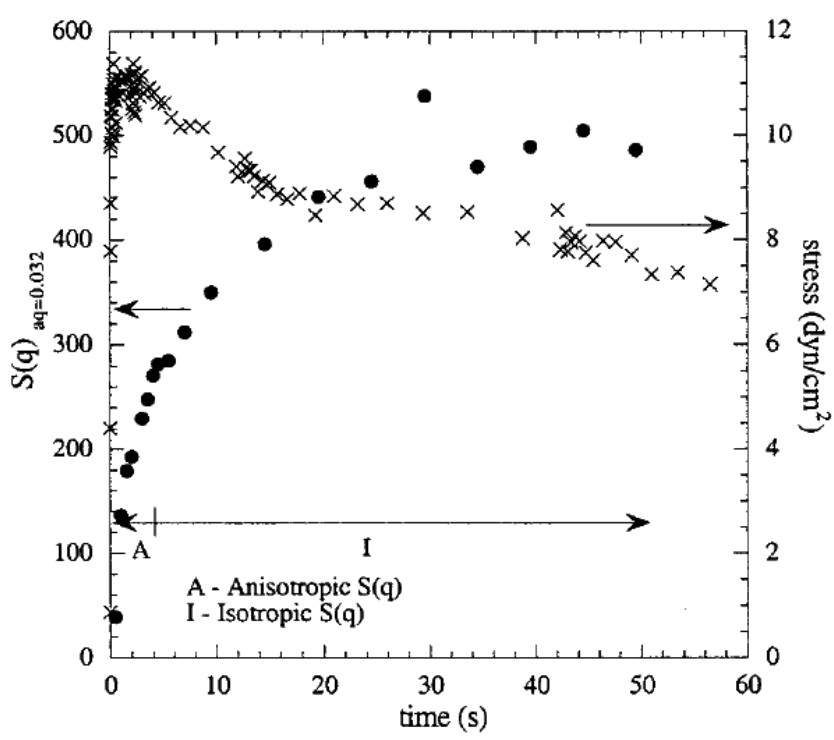

Figure 14. Comparison of the evolution of $S(q)$ at low $q$ with the stress evolution of organophilic silica-hexadecane at $\phi=0.035$ at $3 \mathrm{~s}^{-1}$ (reprinted with permission from Varadan and Solomon (2001), (C) (2001) American Chemcial Society).

For experiments with thixotropic clay dispersions using both neutron and light scattering, it was concluded that the larger length scales are the pertinent ones for rheological properties (Pignon et al 1997). Experiments on better defined model colloids consisting of thermoreversible adhesive spheres, where gelation can be induced by variation of the temperature, also reveal a pronounced butterfly pattern that progressively develops as the material is subjected to flow (Verduin et al 1996, Varadan and Solomon 2001). Figure 14 gives a comparison of the time evolution of the enhancement of $S(\mathbf{q})$ at low $q$ with the stress evolution (Varadan and Solomon 2001), demonstrating the link between anisotropy development and the evolution of the stress.

Video microscopy experiments of 2D particulate monolayers were used to gain insight into the mechanism of the butterfly scattering patterns and the associated microstructural anisotropy (Hoekstra et al 2003). The 2D experiments provide access to the shear-induced microstructure in the $\mathbf{v}-\nabla \mathbf{v}$ plane. Examples of the flow-induced microstructure and its FFT image are shown in figure 15. Anisotropy was shown to exist on both large and local scales, in line with the SALS and SANS results on 3D systems discussed above. 


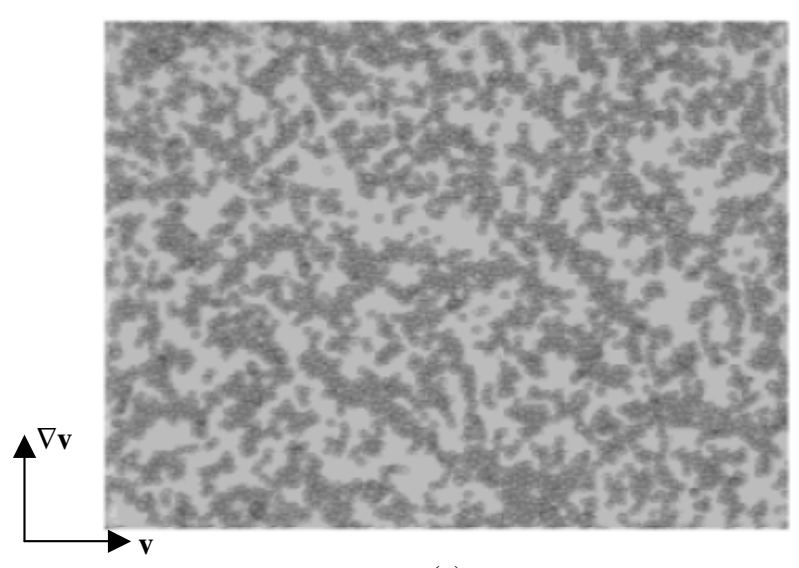

(a)

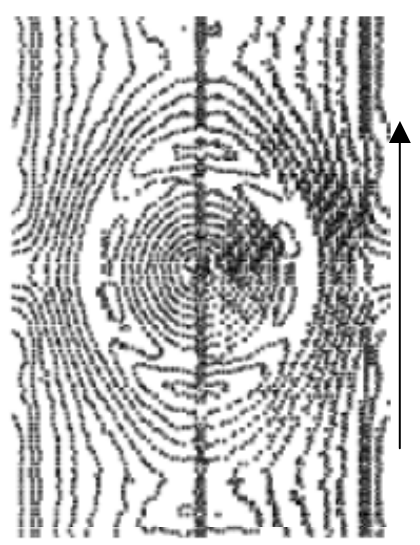

(b)

Figure 15. (a) Microscopy image (snapshot) and (b) isocontour plot of a 2D FFT (averaged over 25 microscopic images) as observed during steady-state flow of micrometre-sized polystyrene spheres at a surface coverage of 0.33 , a shear rate of $0.098 \mathrm{~s}^{-1}$, and a sodiumdodecylsulphate concentration of $1 \mathrm{mM}$. The arrow indicates the velocity direction (reprinted with permission from Hoekstra et al (2003), (c) (2003) American Chemical Society).

To summarize the previous paragraphs, we note the following general features of long length scale scattering anisotropy in colloidal suspensions with attractive interactions.

(1) In many kinds of aggregated suspensions, above a critical shear rate and at sufficiently low scattering vectors, butterfly patterns in the flow-vorticity scattering plane develop with orientation in the flow direction.

(2) The observed scattering is broadly consistent with the physical picture of flocs oriented along the vorticity axis. Spatial reorganization leading to an enhanced separation of these flocs in the flow direction leads to concentration fluctuation enhancement along this axis.

(3) Although the butterfly anisotropic scattering is observed at small scattering vector, additional internal anisotropy of the flocs is also observed at high $q$ in some cases.

(4) The kinetics of the scattering does not reveal transient evolution of a characteristic wavevector of the structure.

(5) There appears to be a correlation between the anisotropic scattering and the rheological response of the suspensions, as is shown in figure 14.

The 2D video microscopy measurements by Hoekstra et al (2003) also provide insight into the mechanism by which these general features develop. The experiments show a directional dependence to break-up and aggregation phenomena during shear flow as shown in figure 16(a); larger objects are mainly formed along the compressional axis of the flow field. When two smaller flocs interlock, they obtain a common rotation speed. These larger flocs remain stable as long as their major axis is oriented in the quadrants following the compressional axis. Only upon reaching the extensional axis do flocs break up again. Because of the rotational component of the shear flow, these break-up and aggregation phenomena occur continually. Together, they yield an anisotropic microstructure. This qualitative mechanism is corroborated by the absence of anisotropy in irrotational extensional flow (Hoekstra et al 2003). It is also in agreement with a gradual enhancement of the local scale inhomogeneity, as exemplified, for example, by a gradual appearance of the butterfly scattering pattern observed in transient studies (Varadan and Solomon 2001) rather than a growth of the scattering pattern from large to small scattering vectors as was the case in phase separating polymer solutions. 

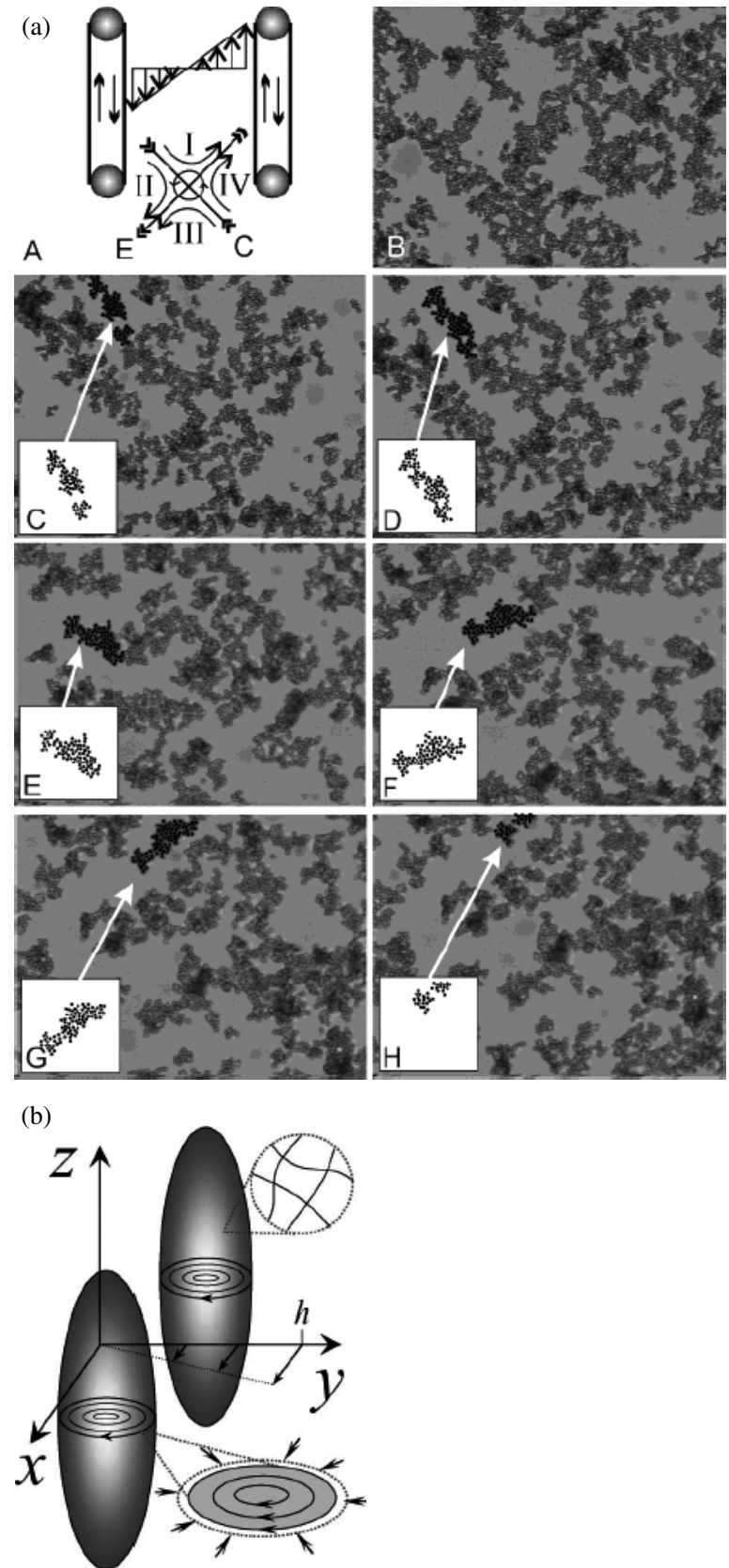

Figure 16. Comparison of the different mechanisms suggested explaining anisotropy development in $2 \mathrm{D}$ suspensions and vorticity orientation in $3 \mathrm{D}$ systems. (a) Apparatus and the flow field showing the compressional (C) and extensional (E) axis of the flow field; and a sequence of flow aggregation (B-D), rotation $(\mathrm{E}-\mathrm{F})$ and break-up $(\mathrm{G}-\mathrm{H})$. All images refer to a 2D suspension with surface coverage of 0.33 and $0.4 \mathrm{M} \mathrm{CaCl} 2$ added to the aqueous phase (reprinted with permission from Hoekstra et al (2003), (C) (2003) American Chemical Society). (b) Cartoon depicting the shear response of the compressible clusters, where internal streamlines are in tension due to elastic forces (reprinted with permission of Lin-Gibson et al (2004), (c) (2004) by the American Physical Society). 
Alternatively, Hobbie et al (2004a) argue that a wide range of butterfly scattering patterns (from emulsions to physical gels of nanoclays in polymer solutions) arise as a consequence of an elastic instability. They suggest that the roller-like organization underlying the butterfly pattern is a characteristic of all soft viscoelastic fluids, dispersed in a less viscoelastic fluid at significant volume fraction. In Montesi et al (2004), confinement effects are an important consideration. The sketch in figure 16(b) depicts the proposed mechanism: internal viscoelastic forces related to the first normal stress differences create 'hoop' stresses, which lead to objects elongated in the vorticity direction. It is not clear at present how this mechanism would apply to essentially inelastic aggregates present in weakly aggregated suspensions, although bending moments known to exist in fractal clusters could play a role.

Although the qualitative mechanisms for anisotropy development of Hoekstra et al (2003) and Lin-Gibson et al (2004) are not mutually exclusive, they differ in several aspects, which should motivate further work. First, comparison of scattering data from multiple systems in the $\mathbf{v}-\nabla \mathbf{v}$ plane is warranted. Second, the sequence of break-up and aggregation processes discovered by Hoekstra et al in 2D suspensions should be sought in other systems. Third, the relationship between anisotropic butterfly scattering and negative first normal stress differences should be more clearly elucidated. Finally, the effect of confinement on large-scale anisotropic structures of aggregated colloidal particles should be compared to results available for nanotube suspensions and attractive emulsions.

\subsection{Unresolved issues and challenges}

A full microstructural picture of flowing weakly aggregated suspensions is slowly emerging. Although mechanisms for the development of large-scale inhomogeneity have been suggested, a full microstructural hierarchy of the structure factor in all directions is lacking. Most scattering observations have focused on probing the structure in the $\mathbf{v}-\omega$ plane, whereas the structure in the $\mathbf{v}-\nabla \mathbf{v}$ plane is needed to obtain a full microstructural understanding. Experiments on 2D suspensions have been carried out in that respect (Hoekstra et al 2003), but it remains to be verified how the observed phenomena can be extrapolated to 3D systems. CSLM measurements on flowing systems (Varadan and Solomon 2003b) are a promising alternative to SALS measurements from the vorticity plane. More complex flow situations, such as squeeze flow, can result in the formation of voids (at phi $=0.26$ ) and cracks (at phi $=0.40$ ) that are both macroscopic in size and characteristic of inhomogeneous strain (Varadan and Solomon 2003b). Toward this end, the possibility of shear-banding or locally inhomogeneous flow in relation to microstructure development needs to be addressed (Vermant 2001, Salmon et al 2003).

A major future challenge is to incorporate the microstructural features discussed here into constitutive descriptions of colloidal gel rheology, which can also predict the nonlinear rheology relevant to processing operations involving colloids. Currently, the link between gel microstructure and rheology has only been described in qualitative terms. The use of optical tweezers to establish the link between micro-mechanical properties and aggregate structure has been suggested as an innovative approach to this problem (Furst 2003, Pantina and Furst 2004).

\section{Suspensions of particles dispersed in viscoelastic media}

\subsection{Introduction and relevance}

In many industrial operations the processed materials consist of particles dispersed in rheologically complex fluids. Common examples are coating and printing operations, as 


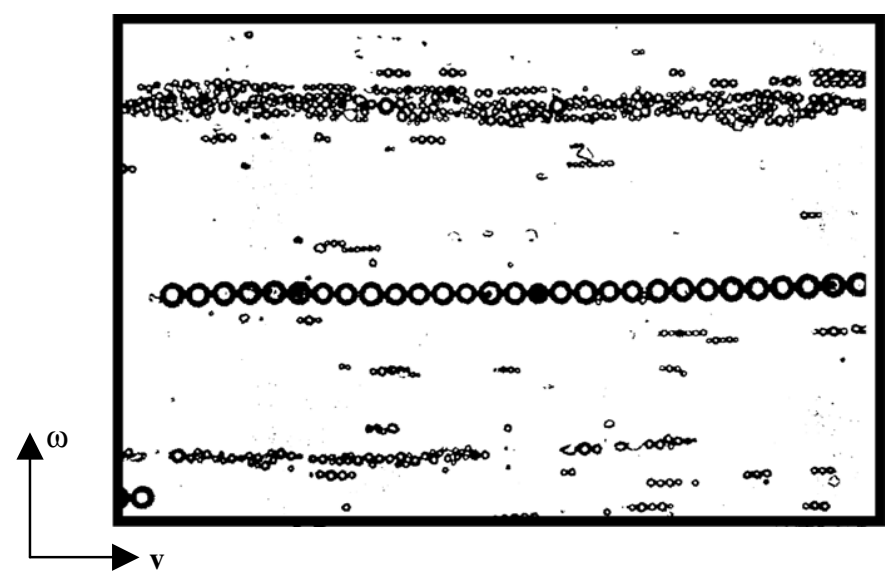

Figure 17. Alignment and segregation effects in suspensions of spheres suspended in a viscoelastic polyacrylamide solution displaying a pronounced alignment and segregation according to particle size when observed in the $\mathbf{v}-\omega$ plane (adapted from Giesekus (1978)). The arrow indicates the flow direction.

well as the processing of filled polymers in general and of nanocomposites in particular (Vaia and Giannelis 2001). Adding particles to a nonlinear viscoelastic fluid, such as a polymer, can considerably increase the rheological complexity of the system (Metzner 1985, Ohl and Gleissle 1993). The spatial distribution of particles plays a dominant role in this respect, as exemplified by particle aggregation or especially flow-induced alignment, which is even observed in dilute suspensions. There is no indication that the same phenomenon occurs for particles dispersed in Newtonian fluids.

\subsection{Effects of flow on particles dispersed in viscoelastic liquids}

Michele et al (1977) subjected dilute suspensions of macroscopic $(60 \mu \mathrm{m})$ glass spheres in a highly viscoelastic polymer solution to rapid oscillatory shearing flows. The resulting microstructures in experiments on polydisperse spheres by Giesekus (1978) are shown in figure 17. Necklaces of particles can clearly be observed. Giesekus (1978) further demonstrated that size segregation effects could be imparted to a bimodal suspension. Recently, Lyon et al (2001) verified the earlier results on dilute suspensions and also reported the formation of particle strings in steady-shear flow for moderately concentrated suspensions with a corresponding quantitative reduction in the shear stress. This study thus confirmed the relevance of the microstructural changes for the rheological properties of suspensions in polymer systems. The physical origin of the particle alignment is associated with normal stress effects (Giesekus 1978). It has even been proposed that a suspending fluid Weissenberg number (here taken as the ratio of the first normal stress difference over the shear stress) of ten for the suspending fluid is necessary for the onset of the necklace formation (Michele et al 1977, Lyon et al 2001). Recently, Scirocco et al (2004) have further investigated the effect of the suspending fluid on the flow-induced alignment. The alignment was studied by means of video microscopy and SALS. Although alignment was observed, the onset of string formation was shown not to be governed by a critical Weissenberg number as assumed earlier. Interestingly, no alignment could be induced in constant viscosity, high elasticity Boger fluids even for a Weissenberg number of 260. A wide range of Weissenberg numbers for the onset of alignment was observed for shear-thinning viscoelastic fluids. 


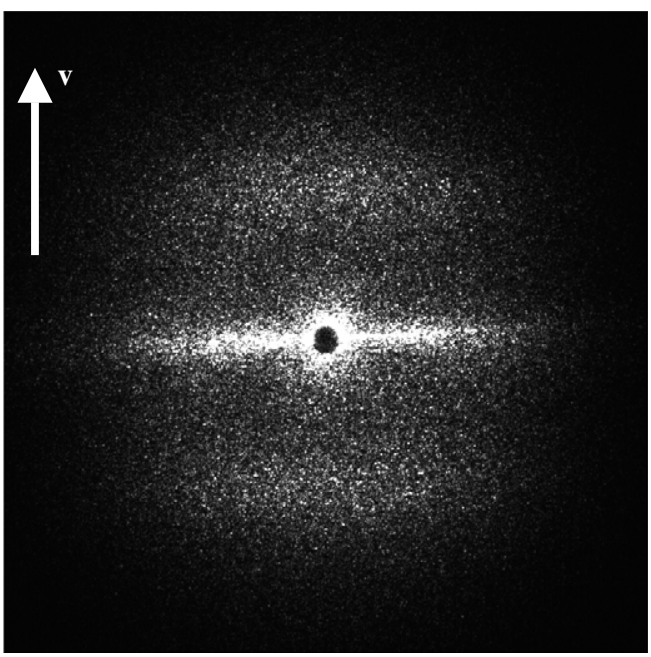

Figure 18. SALS pattern observed under steady state for a suspension of $0.8 \%(\mathrm{v} / \mathrm{v})$ polystrene in a viscoelastic solution of hydroxypropylcellulose in water sheared at $50 \mathrm{~s}^{-1}$ for $3600 \mathrm{~s}$, corresponding to an aligned array of particles with well-defined interparticle spacings. The arrow indicates the flow direction; v- $\omega$ plane (reprinted from Scirocco et al (2004), (C) (2004) with permission from Elsevier).

For a system with pronounced alignment, SALS measurements showed very regular interparticle spacing, as shown in figure 18. The interparticle distance can be estimated from Bragg's law, and it was found to be of the order of $1 \mu \mathrm{m}$ for $3 \mu \mathrm{m}$ spheres. SALS results were also used to demonstrate that the string formation could be very slow, and seems to be, to a first approximation, strain controlled (Scirocco et al 2004). The alignment phenomena are produced at very low volume fractions. This suggests that the hydrodynamic interactions are amplified in viscoelastic fluids, in agreement with recent simulation results (Hwang et al 2004). Dispersing particles into viscoelastic fluids hence might provide an alternative method to produce very ordered structures at low volume fractions. The detailed mechanisms are, however, not yet completely understood.

\section{Conclusions and outlook}

Research focused on understanding the intimate coupling between the flow and the microstructure during the flow of suspensions has greatly advanced through a combination of experimental techniques, theoretical developments and numerical simulations. For fluidlike and ordered suspensions, the effects of flow have been fairly well understood, and even quantitative, predictive links between microstructure and macroscopic properties have been established. For weakly aggregated suspensions and for suspensions of particles in rheologically more complex fluids, more work is needed to develop mechanistic understanding. Possibly, new experimental methods such as in situ CSLM microscopy (Varadan and Solomon 2003a, 2003b, Tolpekin et al 2004) and measurements on two-dimensional model suspensions (Stancik et al 2002, 2003, Hoekstra et al 2003) can considerably contribute to this development. For numerical simulations, Brady and co-workers (see for example Brady 2001) have shown that inclusion of hydrodynamic interactions results in a powerful technique that both helps develop understanding of the physical mechanisms involved and leads to first principles prediction of macroscopic properties. The developments of simulation methods that can accommodate either the non-ergodicity of aggregated suspensions or the non-Newtonian nature of the suspending media are difficult but necessary next steps. In the latter case, promising results are already appearing (Hwang et al 2004). 


\section{References}

Ackerson B J 1990 Shear induced order and shear processing of model hard sphere suspensions J. Rheol. 34 553-90

Ackerson B J and Clark N A 1981 Shear-induced melting Phys. Rev. Lett. 46 123-6

Ackerson B J and Clark N A 1984 Shear-induced partial translational ordering of a colloidal solid Phys. Rev. A 30 906-18

Ackerson B J, Hayter J B, Clark N A and Cotter L 1986 Neutron scattering from charge stabilized suspensions J. Chem. Phys. $842344-9$

Ackerson B J and Pusey P N 1988 Shear-induced order in suspensions of hard spheres Phys. Rev. Lett. 61 $1033-6$

Adriani P M and Gast A P 1989 Predictions of birefringence and dichroism of hard-sphere suspensions in combined electric and shear fields J. Chem. Phys. 91 6282-9

Amos R M, Rarity J G, Tapster P R, Shepherd T J and Kitson S C 2000 Fabrication of large-area face-centered-cubic hard-sphere colloidal crystals by shear alignment Phys. Rev. E 61 2929-35

Arora A K and Tata B V R 1998 Interactions, structural ordering and phase transitions in colloidal dispersions Adv. Colloid Interface Sci. 78 49-97

Ashdown S, Markovic I, Ottewil R H, Lindner P, Oberthur R C and Rennie A R 1990 Small-angle neutron-scattering studies on ordered polymer colloid dispersions Langmuir 6 303-7

Auer S and Frenkel D 2001 Suppression of crystal nucleation in polydisperse colloids due to increased in the surface free energy Nature $\mathbf{4 1 3} 711-3$

Auer S and Frenkel D 2003 Line tension controls wall-induced crystal nucleation in hard-sphere colloids Phys. Rev. Lett. 91015703

Ball R C and Melrose J R 1995 Lubrication breakdown in hydrodynamic simulations of concentrated colloids Adv. Colloid Interface Sci. 59 19-30

Banchio A J and Brady J F 2003 Accelerated Stokesian dynamics: Brownian motion J. Chem. Phys. 118 10323-32

Barnes H A 1997 Thixotropy-a review J. Non-Newton. Fluid Mech. 70 1-33

Bastide J, Leibler L and Prost J 1990 Scattering by deformed swollen gels-butterfly isointensity patterns Macromolecules 23 1821-5

Belzung B et al 2000 Flow-induced anisotropy in mixtures of associative polymers and latex particles $J$. Colloid Interface Sci. 224 179-87

Bender J W and Wagner N J 1995 Optical measurement of the contributions of colloidal forces to the rheology of concentrated suspensions J. Colloid Interface Sci. 172 171-84

Bender J W and Wagner N J 1996 Reversible shear thickening in monodisperse and bidisperse colloidal dispersions J. Rheol. 40 899-916

Bergenholtz J, Brady J F and Vicic M 2002 The non-Newtonian rheology of dilute colloidal suspensions J. Fluid Mech. 456 239-75

Biehl R and Palberg T 2004 Real space and Fourier microscopy of colloidal suspensions confined to a parallel plate geometry Rev. Sci. Instrum. 75 906-14

Bolhuis P G, Frenkel D, Mau S-C and Huse D A 1997 Entropy difference between crystal phases Nature 388 235-6

Boue F and Lindner P 1994 Semidilute polymer solutions under shear Europhys. Lett. 25 421-7

Brady J F 1996 Model hard-sphere dispersions: statistical mechanical theory, simulations, and experiments Curr. Opin. Colloid Interface Sci. $1472-8$

Brady J F 2001 Computer simulation of viscous suspensions Chem. Eng. Sci. 56 2921-6

Brady J F and Bossis G 1988 Stokesian dynamics Annu. Rev. Fluid Mech. 20 111-57

Buscall R, McGowan I J, Mills P D A, Stewart R F, Sutton D, White L R and Yates G E 1987 The rheology of strongly-flocculated suspensions J. Non-Newton. Fluid Mech. 24 183-202

Buscall R, Mills P D A, Goodwin J W and Lawson D W 1988 Scaling behaviour of the rheology of aggregate networks formed from colloidal particles J. Chem. Soc. Faraday Trans. $1844249-60$

Bushell G C, Yan Y D, Woodfield D, Raper J and Amal R 2002 On techniques for the measurement of the mass fractal dimension of aggregates Adv. Colloid Interface Sci. 95 1-50

Butera R J, Wolfe M S, Bender J and Wagner N J 1996 Formation of a highly ordered colloidal microstructure upon flow cessation from high shear rates Phys. Rev. Lett. 77 2117-20

Butler S and Harrowell P 1995 The shear induced disordering transition in a colloidal crystal: nonequilibrium Brownian dynamic simulations J. Chem. Phys. 103 4653-71

Butler S and Harrowell P 2002 Factors determining crystal-liquid coexistence under shear Nature 415 1008-11

Carpineti M and Giglio M 1992 Spinodal-type dynamics in fractal aggregation of colloidal clusters Phys. Rev. Lett. $683327-30$

Catherall A A, Melrose J R and Ball R C 2000 Shear thickening and order-disorder effects in concentrated colloids at high shear rates J. Rheol. $441-25$

Chen L B, Ackerson B J and Zukoski C F 1994a Rheological consequences of microstructural transitions in colloidal crystals J. Rheol. 38 193-216 
Chen L B, Chow M K, Ackerson B J and Zukoski C F 1994b Rheological and microstructural transitions in colloidal crystals Langmuir $\mathbf{1 0} 2817-29$

Chen D H and Doi M 1999 Microstructure and viscosity of aggregating colloids under strong shearing force J. Colloid Interface Sci. 212 286-92

Chen M and Russel W B 1991 Characteristics of flocculated silica dispersions J. Colloid Interface Sci. $141564-77$

Chen L B, Zukoski C F, Ackerson B J, Hanley H J M, Straty G C, Barker J and Glinka C J 1992 Stuctural changes and orientational order in a sheared colloidal suspension Phys. Rev. Lett. 69 688-91

Choi G N and Krieger I M 1986 Rheological studies on sterically stabilized model dispersions of uniform colloidal spheres. 2. Steady-shear viscosity J. Colloid Interface Sci. 113 101-13

Clarke S M, Rennie A R and Ottewill R H 1997 Stacking of hexagonal layers of colloidal particles: study by small-angle neutron scattering Langmuir 13 1964-9

Dawson K A 2002 The glass paradigm for colloidal glasses, gels and other arrested states driven by attractive interactions Curr. Opin. Colloid Interface Sci. 7 218-27

DeGroot J V Jr, Macosko C W, Kume T and Hashimoto T 1994 Flow-induced anisotropic SALS in silica-filled PDMS liquids J. Colloid Interface Sci. 166 404-13

D’Haene P, Mewis J and Fuller G G 1993 Scattering dichroism measurements of flow-induced structures of a shearthickening suspension J. Colloid Interface Sci. $156350-8$

Dhont J K G 1989 On the distortion of the static structure factor of colloidal fluids in shear-flow J. Fluid Mech. 204 421-31

Dhont J K G 1996 An Introduction to Dynamics of Colloids (Amsterdam: Elsevier)

Dickinson E 2000 Structure and rheology of simulated gels formed from aggregated colloidal particles J. Colloid Interface Sci. 225 2-15

Dux C, Musa S, Reus V, Versmold H, Schwahn D and Lindner P 1998 Small angle neutron scattering experiments from colloidal dispersions at rest and under sheared conditions J. Chem. Phys. 109 2556-61

Eiser E, Molino F, Forte G and Pithon X 2000 Flow in micellar cubic crystals Rheol. Acta 39 201-8

Elliot M S, Bristol B T F and Poon W C K 1997 Direct measurement of stacking disorder in hard-sphere colloidal crystals Physica A 235 216-23

Elliott S L, Butera R J, Hanus L H and Wagner N J 2003 Fundamentals of aggregation in concentrated dispersions: fiber-optic quasielastic light scattering and linear viscoelastic measurements Faraday Discuss. 123 369-83

Foss D R and Brady J F 2000 Structure, diffusion and rheology of Brownian suspensions by Stokesian dynamics simulation J. Fluid Mech. 407 167-200

Fuller G G 1995 Optical Rheometry of Complex Fluids (New York: Oxford University Press)

Furst E M 2003 Interactions, structure, and microscopic response: complex fluid rheology using laser tweezers Soft Mater. 1 167-85

Gasser U, Weeks E R, Schofield A, Pusey P N and Weitz D A 2001 Real-space imaging of nucleation and growth in colloidal crystallization Science 292 258-62

Gast A P and Russel W B 1998 Simple ordering in complex fluids-colloidal particles suspended in solution provide intriguing models for studying phase transitions Phys. Today 51 (12) 24-30

Giesekus H 1978 Die Bewegung von Teilchen in Strömungen nicht-newtonscher Flüssigkeiten Z. Angew. Math. Mech. 58 T26-37

Grant M C and Russel W B 1993 Volume fraction dependence of elastic-moduli and transition temperatures for colloidal silica gels Phys. Rev. E 47 2606-14

Gratson G M, Mingjie X and Lewis J A 2004 Direct writing of three-dimensional webs Nature 428 386-9

Gray J J and Bonnecaze R T 1998 Rheology and dynamics of sheared arrays of colloidal particles J. Rheol. $421121-51$

Guinier A 1963 X-Ray Diffraction (New York: Dover)

Harland J L and van Megen W 1997 Crystallization kinetics of suspensions of hard colloidal spheres Phys. Rev. E 55 3054-67

Hashimoto T and Kume T 1992 Butterfly light-scattering pattern in shear-enhanced concentration fluctuations in polymer-solutions and anomaly at high shear J. Phys. Soc. Japan 61 1839-43

Haw M D, Poon W C K and Pusey P N 1998 Direct observation of oscillatory-shear-induced order in colloidal suspensions Phys. Rev. E 57 6859-64

Helfland E and Fredrickson G 1989 Large fluctuations in polymer solutions under shear Phys. Rev. Lett. 62 2468-71

Heni M and Löwen H 2000 Surface freezing on patterned substrates Phys. Rev. Lett. 85 3668-71

Hess S and Hanley H J M 1983a Shear-flow induced distortion of the radial-distribution function, est of a model kinetic-equation in the non-linear flow regime Physica A 118 444-9

Hess S and Hanley H J M 1983b Stokes-Maxwell relations for the distorted fluid microstructure Phys. Lett. A 98 $35-8$ 
Hiemenz P C and Rajagopalan R 1997 Principles of Colloid and Surface Chemistry 3rd edn (New York: Dekker)

Hobbie E K and Migler K B 1999 Vorticity elongation in polymeric emulsions Phys. Rev. Lett. 82 5393-6

Hobbie E K et al 2002 Shear-induced structure in polymer blends with viscoelastic asymmetry J. Chem. Phys. 117 6350-9

Hobbie E K et al 2004 Ubiquity of domain patterns in sheared viscoelastic fluids Phys. Rev. E 69061503

Hoekstra H, Vermant J, Mewis J and Fuller G G 2003 Flow-induced anisotropy and reversible aggregation in twodimensional suspensions Langmuir 19 9134-41

Hoffman R L 1972 Discontinuous and dilatant viscosity behavior in concentrated suspensions. I. Observation of a flow instability J. Rheol. 16 155-73

Hoffmann G P and Lowen H 2001 Freezing and melting criteria in non-equilibrium J. Phys.: Condens. Matter 13 9197-206

Hoogenboom J P, Derks D, Vergeer P and van Blaaderen A 2002 Stacking faults in colloidal crystals grown by sedimentation J. Chem. Phys. 117 11320-8

Hwang W R, Hulsen M A and Meijer H E H 2004 Direct simulations of particle suspensions in a viscoelastic fluid in sliding bi-periodic frames J. Non-Newton. Fluid Mech. 121 15-33

Irvine D J, Stachowiak A and Jain S 2003 Engineering biomaterials for control of immune cell functions Mater. Sci. Forum 426 3213-8

Jiang P, Bertone J F, Hwang K S and Colvin V L 1999 Single-crystal colloidal multilayers of controlled thickness Chem. Mater. 11 2132-40

Kadoma I A and van Egmond J W 1997 Shear-enhanced orientation and concentration fluctuations in wormlike micelles: effect of salt Langmuir 13 4551-61

Kegel W K and Dhont J K G 2000 'Aging' of the structure of crystals of hard colloidal spheres J. Chem. Phys. 112 3431-6

Kremer K, Robbins M O and Grest G S 1986 Phase diagram of Yukawa systems: model for charge-stabilized colloids Phys. Rev. Lett. 57 2694-7

Krieger I M and Choi G N 1982 Rheology of sterically stabilized colloidal dispersions J. Rheol. 26 577-8

Larson R G 1999 Rheology and Structure of Complex Fluids (Oxford: Oxford University Press)

Laun H M, Bung R, Hess S, Loose W, Hess O, Hahn K, Hadicke E, Hingmann R, Schmidt F and Lindner P 1992 Rheological and small angle neutron scattering investigation of shear-induced particle structures of concentrated polymer dispersions submitted to plane Poiseuille and Couette flow J. Rheol. 36 743-87

Lewis J A 2000 Colloidal processing of ceramics J. Am. Ceram. Soc. 83 2341-59

Lin-Gibson S, Pathak J A, Grulke E A and Hobbie E K 2004 Elastic flow instability in nanotube suspensions Phys. Rev. Lett. 92048302

Loose W and Ackerson B J 1994 Model calculations for the analysis of scattering data from layered structures J. Chem. Phys. 101 7211-20

Lowen H 2004 The marriage of electrostatics and hydrodynamics: simulating the dynamics of charged colloids J. Phys.: Condens. Matter 16 V7-9

Lyon M K, Mead D W, Elliot R E and Leal G L 2001 Structure formation in moderately concentrated viscoelastic suspensions in simple shear flow J. Rheol. $45881-90$

Maranzano B J and Wagner N J 2002 Flow-small angle neutron scattering measurements of colloidal dispersion microstructure evolution through the shear thickening transition J. Chem. Phys. 117 10291-302

Melrose J R and Ball R C 2004 Continuous shear thickening transitions in model concentrated colloids-the role of interparticle forces $J$. Rheol. 48 937-60

Mewis J 1979 Thixotropy_general review J. Non-Newton. Fluid Mech. 6 1-20

Mewis J and Biebaut G 2001 Shear thickening in steady and superposition flows: effect of particle interaction forces J. Rheol. 45 799-814

Metzner A B 1985 Rheology of suspensions in polymeric liquids J. Rheol. 29 739-75

Michele J, Pätzold R and Donis R 1977 Alignment and aggregation effect in suspensions of spheres in non-Newtonian media Rheol. Acta 16 317-21

Mitchell P J, Heyes D M and Melrose J R 1995 Brownian-dynamics simulations of model stabilised colloidal dispersions under shear J. Chem. Soc. Faraday Trans. 91 1975-89

Montesi A, Pena A A and Pasquali M 2004 Vorticity alignment and negative normal stresses in sheared attractive emulsions Phys. Rev. Lett. 92058303

Moses E, Kume T and Hashimoto T 1994 Shear microscopy of the butterfly pattern in polymer mixtures Phys. Rev. Lett. 72 2037-40

Ohl N and Gleissle W 1993 The characterization of the steady-state shear and normal stress functions of highly concentrated suspensions formulated with viscoelastic liquids J. Rheol. 37 381-406 
Panine P, Narayanan T, Vermant J and Mewis J 2002 Structure and rheology during shear-induced crystallization of a latex suspension Phys. Rev. E 66022401

Pantina J P and Furst E M 2004 Directed assembly and rupture mechanics of colloidal aggregates Langmuir 20 3940-6

Pham K N, Puertas A M, Bergenholtz J, Egelhaaf S U, Moussaid A, Pusey P N, Schofield A, Cates M E, Fuchs M and Poon W C K 2002 Multiple glassy states in a simple model system Science 296 104-6

Pignon F, Magnin A and Piau J-M 1997 Butterfly light scattering pattern and rheology of a sheared thixotropic clay gel Phys. Rev. Lett. 79 4689-92

Potanin A A, Derooij R, Vandenende D and Mellema J 1995 Microrheological modeling of weakly aggregated dispersions J. Chem. Phys. 102 5845-53

Prasad V, Trappe V, Dinsmore A D, Segre P N, Cipelletti L and Weitz D A 2003 Universal features of the fluid to solid transition for attractive colloidal particles Faraday Discuss. 123 1-12

Pronk S and Frenkel D 1999 Can stacking faults in hard-sphere crystals anneal out spontaneously? J. Chem. Phys. 110 4589-92

Pusey P N and van Megen W 1986 Phase behaviour of concentrated suspensions of nearly hard colloidal spheres Nature 320 340-2

Pusey P N, van Megen W, Bartlett P, Ackerson B J, Rarity J G and Underwood S M 1989 Structure of crystals of hard colloidal spheres Phys. Rev. Lett. $632753-6$

Rastogi S R, Wagner N J and Lustig S R 1996a Rheology, self-diffusion, and microstructure of charged colloids under simple shear by massively parallel nonequilibrium Brownian dynamics J. Chem. Phys. 104 9234-48

Rastogi S R, Wagner N J and Lustig S R 1996b Microstructure and rheology of polydisperse, charged suspensions J. Chem. Phys. 104 9249-58

Rueb C J and Zukoski C F 1997 Elastic properties of colloidal gels J. Rheol. 41 197-218

Russel W B and Gast A P 1986 Nonequilibrium statistical-mechanics of concentrated colloidal dispersions-hardspheres in weak flows J. Chem. Phys. 84 1815-26

Russel W B, Saville D A and Schowalter W R 1989 Colloidal Dispersions (Cambridge: Cambridge University Press)

Salmon J B, Becu L, Manneville S and Colin A 2003 Towards local rheology of emulsions under Couette flow using dynamic light scattering Eur. Phys. J. 10 209-21

Sawada T, Suzuki Y, Toyotama A and Iyi N 2001 Quick fabrication of gigantic single-crystalline colloidal crystals for photonic crystal applications Japan. J. Appl. Phys. 40 L1226-8

Schaefer D W, Martin J E, Wiltzius P and Cannel D S 1984 Fractal geometry of colloidal aggregates Phys. Rev. Lett. 52 2371-4

Schubert B A et al 2004 Shear-induced phase separation in solutions of wormlike micelles Langmuir 20 3564-73

Scirocco R, Vermant J and Mewis J 2004 Effect of the viscoelasticity of the suspending fluid on structure formation in suspensions J. Non-Newton. Fluid Mech. 117 183-92

Shah S A, Chen Y L, Ramakrishnan S, Schweizer K S and Zukoski C F 2003 Microstructure of dense colloid-polymer suspensions and gels J. Phys.: Condens. Matter 15 4751-78

Shih W H, Shih W Y, Kim S I, Liu J and Aksay I A 1990 Scaling behaviour of the elastic properties of colloidal gels Phys. Rev. A 42 4772-9

Sierou A and Brady J F 2002 Rheology and microstructure in concentrated noncolloidal suspensions J. Rheol. 46 1031-56

Silbert L E, Farr R S, Melrose J R and Ball R C 1999a Stress distributions in flowing aggregated colloidal suspensions J. Chem. Phys. 111 4780-9

Silbert L E and Melrose J R 1999 The rheology and microstructure of concentrated, aggregated colloids J. Rheol. 43 $673-700$

Silbert L E, Melrose J R and Ball R C 1999b A structural analysis of concentrated, aggregated colloids under flow Mol. Phys. 96 1667-75

Sirota E B, Ou-Yang H D, Sinha S K, Chaikin P M, Axe J D and Fujii Y 1989 Complete phase diagram of a charged colloidal system: a synchrotron X-ray scattering study Phys. Rev. Lett. 62 1524-7

Solomon M J, Almusallam A S, Seefeldt K F, Somwangthanaroj A and Varadan P 2001 Rheology of polypropylene/clay hybrid materials Macromolecules 34 1864-72

Sonntag R C and Russel W B 1987 Elastic properties of flocculated networks J. Colloid Interface Sci. 116 485-9

Stancik E J, Gavranovic G T, Widenbrant M J O, Laschitsch A T, Vermant J and Fuller G G 2003 Structure and dynamics of particle monolayers at a liquid-liquid interface subjected to shear flow. 3 Faraday Discuss. 123 $145-56$

Stancik E J, Hawkinson A L, Vermant J and Fuller G G 2004 Dynamic transitions and oscillatory melting of a two-dimensional crystal subjected to shear flow J. Rheol. 48 159-73

Stancik E J, Widenbrant M J O, Laschitsch A T, Vermant J and Fuller G G 2002 Structure and dynamics of particle monolayers at a liquid-liquid interface subjected to extensional flow Langmuir 18 4372-8 
Stevens M J and Robbins M O 1993 Simulations of shear-induced melting and ordering Phys. Rev. E 48 $3778-92$

Stieger M and Richtering W 2003 Shear-induced phase separation in aqueous polymer solutions: temperature-sensitive microgels and linear polymer chains Macromolecules 36 8811-8

Terray A, Oakey J and Marr D W M 2002 Microfluidic control using colloidal devices Science 296 1841-4

Tolpekin V A, Duits M H G, van den Ende D and Mellema J 2004 Aggregation and breakup of colloidal particle aggregates in shear flow, studied with video microscopy Langmuir 20 2614-27

Torres F E, Russel W B and Schowalter W R 1991 Flow structure and growth kinetics for rapid shear coagulations of polystyrene colloids J. Colloid Interface Sci. 142 554-74

Trappe V and Weitz D A 2000 Scaling of the viscoelasticity of weakly attractive particles Phys. Rev. Lett. 85 449-52

Vaia R and Giannelis E P 2001 Polymer nanocomposites Mater. Res. Soc. Bull. 26 394-401

van Blaaderen A 2004 Colloids under external control Mater. Res. Soc. Bull. 29 85-90

van Blaaderen A, Ruel R and Wiltzius P 1997 Template-directed colloidal crystallization Nature 385 321-4

Vanderaerschot E and Mewis J 1992 Equilibrium properties of reversibly flocculated dispersions Colloids Surf. 69 $15-22$

Vandeven T G M and Mason S G 1977a Microrheology of colloidal dispersions: effect of shear on perikinetic doublet formation Colloid Polym. Sci. 255 794-804

Vandeven T G M and Mason S G 1977b Microrheology of colloidal dispersions: orthokinetic formation of spheres Colloid Polym. Sci. 255 468-79

Van Egmond J W 1997 Effect of stress-structure coupling on the rheology of complex fluids: poor polymer solutions Macromolecules 30 8045-57

Van Egmond J W, Werner D E and Fuller G G 1992 Time-dependent small angle light scattering of shear induced concentration fluctuations in polymer solutions J. Chem. Phys. $967742-57$

Varadan P and Solomon M J 2001 Shear-induced microstructural evolution of a thermoreversible colloidal gel Langmuir 17 2918-29

Varadan P and Solomon M J 2003a Direct visualization of long range structural heterogeneity in colloidal gels Langmuir 19 509-12

Varadan P and Solomon M J 2003b Direct visualization of flow-induced microstructure in dense colloidal gels by confocal laser scanning microscopy J. Rheol. 47 943-68

Verduin H, de Gans B J and Dhont J K G 1996 Shear induced structural changes in a gel-forming suspensions studied by light scattering and rheology Langmuir 12 2947-55

Vermant J 2001 Large-scale structures in sheared colloidal dispersions Curr. Opin. Colloid Interface Sci. $6489-95$

Vermant J, Raynaud L, Mewis J, Ernst B and Fuller G G 1999 Large-scale bundle ordering in sterically stabilized latices J. Colloid Interface Sci. $211221-9$

Versmold H, Musa S, Dux C, Lindner P and Urban V 2001 Shear-induced structure in concentrated dispersions: small angle synchrotron X-ray and neutron scattering Langmuir 17 6812-5

Vlasov Y A, Yao N and Norris D J 1999 Synthesis of photonic crystals for optical wavelengths from semiconductor quantum dots Adv. Mater. 11 165-9

Wagner N J and Ackerson B J 1992 Analysis of nonequilibrium structures of shearing colloidal suspensions J. Chem. Phys. 97 1473-81

Wagner N J, Fuller G G and Russel W B 1988 The dichroism and birefringence of a hard-sphere suspension under shear J. Chem. Phys. 89 1580-90

Wagner N J and Russel W B 1990 Light-scattering measurements of a hard-sphere suspension under shear Phys. Fluids A 2 491-502

Wheeler E K, Izu P and Fuller G G 1996 Structure and rheology of wormlike micelles Rheol. Acta 35 139-49

Wilson H J and Davis R H 2000 The viscosity of a dilute suspension of rough spheres J. Fluid Mech. 421 339-67

Woodcock L V 1997 Entropy difference between the face-centered cubic and hexagonal close-packed crystal structures Nature 385 141-3

Woutersen A T J M and Dekruif C G 1991 The rheology of adhesive hard-sphere dispersions J. Chem. Phys. 94 5739-50

Woutersen A T J M, May R P and DeKruif C G 1993 The shear-distorted microstructure of adhesive hard-sphere dispersions-a small-angle neutron-scattering study J. Rheol. 37 71-88

Yan Y D and Dhont J K G 1993 Shear-induced structure distortion in nonaqueous dispersions of charged colloidal spheres via light scattering Physica A 198 78-107

Yethiraj A and van Blaaderen A 2003 A colloidal model system with an interaction tunable from hard sphere to soft and dipolar Nature $421513-7$

Zhu J, Li M, Rogers R, Meyer W, Ottewill R H, Space Shuttle Crew, Russel W B and Chaikin P M 1997 Crystallization of hard-sphere colloids in microgravity Nature $\mathbf{3 8 7} 883-5$ 